\begin{tabular}{|c|l|}
\hline Title & Stability of a boundary spike lay er for the Gierer-Meinhardt system \\
\hline Author(s) & Miyamoto, Y asuhito \\
\hline Citation & $\begin{array}{l}\text { European Journal of A pplied Mathematics, 16/4), 467-491 } \\
\text { https://doi.org/40.1017/S0956792505006376 }\end{array}$ \\
\hline Issue Date & 2005-08 23 \\
\hline Doc URL & http://hdl.handle.net/2115/5419 \\
\hline Rights & Copyright $\odot 2005$ Cambridge University Press \\
\hline Type & article \\
\hline File Information & EJAM16.pdf \\
\hline
\end{tabular}

Instructions for use 


\title{
Stability of a boundary spike layer for the Gierer-Meinhardt system
}

\author{
YASUHITO MIYAMOTO \\ Meme Media Laboratory, Hokkaido University: \\ Kita 13 Nishi 8, Kita-ku, Sapporo 060-0813, Japan \\ e-mail: miyamoto@nsc.es.hokudai.ac.jp
}

(Received 7 December 2004; revised 23 August 2005)

\begin{abstract}
We consider the activator-inhibitor Gierer-Meinhardt reaction-diffusion system of biological pattern formation in a closed bounded domain. The existence and stability of a boundary apike-layer solution to the Gierer-Meinhardt model, and it, so-called shadow limit, is analysed. In the limit of small activator diffusivity, together with a large inhibitor diffusivity, an equilibrium boundary spike-layer solution is constructed that concentrates at a non-degenerate critical point $P$ of the boundary. By non-degenerate we mean that every principal curvature of the boundary has a local maximum at $P$, and hence the mean curvature at the boundary has a local maximum at $P$. Rigorous results for the stability of such a boundary spike-layer solution are given.
\end{abstract}

\section{Introduction}

In this paper we are concerned with the existence and the stability of stationary solutions for the multi-dimensional reaction-diffusion system

$$
\begin{gathered}
\frac{\partial A}{\partial t}=\varepsilon^{2} \Delta A-\mu A+\frac{A^{p}}{H^{q}}, \quad \sigma \frac{\partial H}{\partial t}=\frac{1}{\tau} \Delta H-v H+\frac{A^{r}}{H^{s}} \quad \text { in } \Omega \times \mathbb{R}_{+}, \\
\frac{\partial A}{\partial n}=\frac{\partial H}{\partial n}=0 \quad \text { on } \partial \Omega \times \mathbb{R}_{+} .
\end{gathered}
$$

Here $\Omega \subset \mathbb{R}^{N}$ is a bounded domain with smooth boundary $\partial \Omega$, and $\frac{\partial}{\partial n}$ denotes the outer normal derivative. This system (1.1) was proposed by Gierer \& Meinhardt [4] to model biological pattern formation. The unknowns $A=A(x, t)$ and $H=H(x, t)$ denote the concentrations of the biochemicals called an activator and an inhibitor, and $\varepsilon, \tau, \sigma, \mu$ and $v$ are positive constants. Here $\mathbb{R}_{+}:=\{a \mid a>0\}$, and $1<p<((N+2) /(N-2))_{+}$, that, $1<p<+\infty$ for $N=1$ or 2 , and $1<p<(N+2) /(N-2)$ for $N \geqslant 3$. We assume that the exponents $p, q, r$ and $s$ satisfy

$$
p>1, q>0, r>0, s \geqslant 0 \text { and } 0<\frac{p-1}{q}<\frac{r}{s+1} .
$$

To guarantee a certain symmetry of an operator on functional spaces, we will assume throughout this paper that

$$
r=p+1 .
$$


For ease of notation, we define $\gamma$ as

$$
\gamma:=\frac{q r}{p-1}-(s+1)
$$

It is easy to see that $\gamma$ is positive by assumption (1.2).

Numerical simulations of the Gierer-Meinhardt system (1.1) indicate that when $\varepsilon \tau>0$ is small, this system seems to have stable stationary solutions with the property that the activator concentration is localized around a finite number of points in $\bar{\Omega}$. Moreover, the pattern exhibits a "point-condensation phenomenon": as $\varepsilon$ decreases, the activator concentration becomes increasingly localized to narrow regions around some points. As $\varepsilon$ tends to 0 these localized region shrink to a few points where the maximum value of the activator concentration tends to infinity.

There is also a solution where the activator concentration is localized around one point on the boundary of $\Omega$. This steady state is usually called a boundary spike layer.

We define the variables $u$ and $v$ as

$$
u=\left(\frac{\mu^{s+1}}{v^{q}}\right)^{\frac{1}{(p-1) \gamma}} A \text { and } v=\left(\frac{\mu^{r}}{v^{p-1}}\right)^{\frac{1}{(p-1) \gamma}} H
$$

Then $u$ and $v$ satisfy

$$
\begin{gathered}
\frac{\partial u}{\partial t}=\varepsilon^{2} \Delta u-u+\frac{u^{p}}{v^{q}}, \quad \sigma \frac{\partial v}{\partial t}=\frac{1}{\tau} \Delta v-v+\frac{u^{r}}{v^{s}} \quad \text { in } \Omega \times \mathbb{R}_{+}, \\
\frac{\partial u}{\partial n}=\frac{\partial v}{\partial n}=0 \quad \text { on } \partial \Omega \times \mathbb{R}_{+} .
\end{gathered}
$$

The parameters $\varepsilon / \sqrt{\mu},(\mu / v) \sigma, v \tau$ and $\mu t$ are re-labelled with $\varepsilon, \sigma, \tau$ and $t$. We call (1.4) the full system. For reductions from general forms of the Gierer-Meinhardt system, see Ward \& Wei [19].

If $\tau$ tends to zero, then the diffusion coefficient of the second equation of this system tends to infinity. Therefore, we can expect that $v(x, t)$ tends to a spatially homogeneous function $\xi(t)$ which depends only on $t$. Then integrating both sides of the second equation of the system (1.4) over $\Omega$ together with the Neumann boundary condition, we obtain the system

$$
\begin{gathered}
\frac{\partial u}{\partial t}=\varepsilon^{2} \Delta u-u+\frac{u^{p}}{\xi^{q}}, \quad \sigma \frac{d \xi}{d t}=-\xi+\frac{1}{\xi^{s}} \int_{\Omega} u^{r}(y, t) d y \quad \text { in } \Omega \times \mathbb{R}_{+}, \\
\frac{\partial u}{\partial n}=0 \quad \text { on } \partial \Omega \times \mathbb{R}_{+} .
\end{gathered}
$$

Following Nishiura [8], we call the system the shadow system.

$\mathrm{Ni}$ et al. [13] showed that the so-called least-energy pattern, which corresponds to one of the boundary spike layer solutions of the shadow system (1.5), is weakly stable if the condition (A0) holds. Moreover, they showed that the least-energy pattern is stable if the domain is an annulus. The stability of an interior spike equilibrium was also disscussed by several authors $[9,18,19,20]$.

We now make one remark on the technique of the proofs that we use in the paper. In our analysis of the stability of steady state solutions in the shadow system (see subsection 2.3) the key assumption (A0) leads to a certain linear operator that is self-adjoint. For 
the full Gierer-Meinhardt system, without assumption (A0), the linearized operator is not self-adjoint and the analysis is more difficult. In the case of the full system (1.4) complex eigenvalues should be considered. The main technical results of this paper are Lemmas 4.4 and 4.6 dealing with complex eigenvalues.

In the present paper, we prove the stability of the boundary spike layer for the full system (1.4). We prove that there is a constant $\alpha>0$ such that a linearized operator $\left(\mathscr{L}_{\varepsilon, \tau}-\lambda\right)$ of the full system (1.4) at a boundary spike layer is invertible in a certain functional space for all $\lambda \in \Lambda_{\alpha}$, where

$$
\Lambda_{\alpha}:=\{\lambda \in \mathbb{C} \mid \operatorname{Re}(\lambda)>-\alpha\} .
$$

This paper is organized as follows: in $\S 2$ we state some results for a single semilinear elliptic equation (2.1) and formulate main results, namely Theorems A, B and C, which are concerning with the existence of a boundary spike layer of (1.4) (Theorem A) and the stability of a boundary spike layer of the full system (1.4) and of the shadow system (1.5) (Theorems B and C respectively). A brief sketch of the proof of these theorems is given. In $\S 3$ we derive useful equalities which are used thereafter in $\S 4$ and 5 . We also give asymptotics for the eigenvalues of some problems (Lemmas 3.1 and 3.3). In $\S 4$ we consider the invertibility of the linear operator $\left(\mathscr{L}_{\varepsilon, 0}-\lambda\right)$ for $\lambda \in \Lambda_{\alpha}$ and prove Theorems A and C. In $\S 5$ we show that the linear operator $\left(\mathscr{L}_{\varepsilon, \tau}-\lambda\right)$ is close in some sense to the linear operator $\left(\mathscr{L}_{\varepsilon, 0}-\lambda\right)$. Using this fact, we construct the inverse of $\left(\mathscr{L}_{\varepsilon, \tau}-\lambda\right)$ for all $\lambda \in \Lambda_{\alpha}$. This completes the proof of Theorem B. Some conclusion are made in Section 6.

\section{Main results}

\subsection{Main theorems}

Firstly, we define the functional spaces $X, Y$ and $C_{N}^{2+d}$ as

$$
X:=L^{2}(\Omega), \quad Y:=\left\{u(x) \in H^{2}(\Omega) \mid \frac{\partial u}{\partial n}=0 \text { on } \partial \Omega\right\}
$$

and

$$
C_{N}^{2+d}:=\left\{u(x) \in C^{2}(\Omega) \mid u, \frac{\partial u}{\partial x_{j}}, \frac{\partial^{2} u}{\partial x_{j} \partial x_{k}} \in C^{d}(\Omega)(1 \leqslant j, k \leqslant N), \frac{\partial u}{\partial n}=0 \text { on } \partial \Omega\right\} .
$$

Here $x=\left(x_{1}, x_{2}, \cdots, x_{N}\right)$ and $0<d<1$. The functional space $C^{d}(0<d<1)$ is a standard Hölder space consisting of the functions that are uniformly Hölder continuous with exponent $d$ in $\Omega$ [5]. By $\langle\cdot, \cdot\rangle$ we denote the inner product in $X$ as $\langle u, v\rangle:=\int_{\Omega} u(x) v(x) d x$, and $\|u\|_{X}^{2}=\langle u, u\rangle$.

Before we state our main results, we recall some results for the existence of a boundary spike layer for the shadow system (1.5) [15]. Let $\hat{u}(x)=\xi^{-\frac{q}{p-1}} u(x)$; then $(u, \xi) \in Y \times \mathbb{R}$ is a solution for the shadow system (1.5) if and only if $(\hat{u}, \xi) \in Y \times \mathbb{R}$ satisfies

$$
\varepsilon^{2} \Delta \hat{u}-\hat{u}+\hat{u}^{p}=0 \quad \text { in } \Omega, \quad \hat{u}>0 \quad \text { on } \Omega \quad \text { and } \quad \frac{\partial \hat{u}}{\partial n}=0 \quad \text { on } \partial \Omega,
$$

and

$$
\xi^{-\gamma}=\int_{\Omega} \hat{u}^{r} d x
$$

From now on, we assume that $\left(\hat{u}_{\varepsilon}, \xi_{\varepsilon}\right)$ is a solution for (2.1) and (2.2). 
The problem (2.1) has been well-studies, and a number of important results were obtained $[3,6,7,10,11,15]$. Here we recall a simplified version of these results $[3,6]$, which are sufficient for our purpose.

Proposition 2.1 $[15,6,3]$ Let $P_{0} \in \partial \Omega$. Suppose that $P_{0}$ is a critical point of the mean curvature function $H(P)$. Then if $\varepsilon$ is sufficiently small, there exists a solution $\hat{u}_{\varepsilon}$ for $(2.1)$ such that $\hat{u}_{\varepsilon}$ has only one local (and hence, global) maximum point $P_{\varepsilon}$. Moreover, $P_{\varepsilon} \rightarrow P_{0} \in \partial \Omega$ as $\varepsilon \rightarrow 0$. Therefore $\left(\xi_{\varepsilon}^{\frac{q}{p-1}} \hat{u}_{\varepsilon}(x), \xi_{\varepsilon}\right)$ is a boundary spike layer for (1.5).

Wei [15] proved Proposition 2.1 under the condition that $P_{0}$ is a non-degenerate critical point of the mean curvature function $H(P)$. Li [6] has relaxed the non-degeneracy condition. Del Pino et al. [3] proved the existence of the boundary spike layer for semilinear elliptic equations with a general nonlinearity.

Definition 2.2 Let $H(P)$ be a mean curvature at $P \in \partial \Omega$, and let $G(P):=\left(\partial_{j} \partial_{k} H(P)\right)$, where $\partial_{i}(i=1,2, \cdots, N-1)$ denotes the $(N-1)$-tangential derivatives. We will call here the point $P_{0}$ a non-degenerate local maximum point of every principal curvature of $\partial \Omega$ if the matrix $-G\left(P_{0}\right)$ is positive definite.

We are now in a position to state our main results.

Theorem A Suppose that the condition (A0) holds, $N \geqslant 2, P_{0} \in \partial \Omega$ is a non-degenerate local maximum point of every principal curvature of $\partial \Omega$, and $\left(\hat{u}_{\varepsilon}(x), \xi_{\varepsilon}\right)$ is a solution for (2.1) and (2.2) such that the maximum point $P_{\varepsilon}$ of $\hat{u}_{\varepsilon}(x)$ tends to $P_{0}$ as $\varepsilon \rightarrow 0$. Then for any small $\varepsilon>0$, there is a $\tau_{*}=\tau_{*}(\varepsilon)>0$ and an analytic mapping $\tau \mapsto\left(u_{\varepsilon, \tau}(x), v_{\varepsilon, \tau}(x)\right)$ from $\left[0, \tau_{*}\right)$ into $C_{N}^{2}(\Omega) \times C_{N}^{2}(\Omega)$ such that

(i) $\left(u_{\varepsilon, \tau}(x), v_{\varepsilon, \tau}(x)\right)$ is a solution for (1.4), and

(ii) $\left(u_{\varepsilon, 0}(x), v_{\varepsilon, 0}(x)\right)=\left(\xi_{\varepsilon}^{\frac{q}{p-1}} \hat{u}_{\varepsilon}(x), \xi_{\varepsilon}\right)$.

In the case $N=1$, Takagi [14] proved existence of a boundary spike layer for the full system (1.4) and the shadow system (1.5). For $N=2$ or 3, Del Pino et al. [2] proved the existence of a boundary spike layer for the original Gierer-Meinhardt system (1.5) $((p, q, r, s)=(2,1,2,0))$ in the case that the critical point of the mean curvature function is not necessarily non-degenerate.

Throughout the present paper we denote $\left(u_{\varepsilon, \tau}, v_{\varepsilon, \tau}\right)$ and $\left(u_{\varepsilon, 0}, v_{\varepsilon, 0}\right)$ as the boundary spike layer solutions for the full system (1.4) and the shadow system (1.5) respectively. In particular, $\left(u_{\varepsilon, 0}, v_{\varepsilon, 0}\right)=\left(\xi_{\varepsilon}^{\frac{q}{p-1}} \hat{u}_{\varepsilon}, \xi_{\varepsilon}\right)$.

Remark 2.3 Theorem A indicates that $\left(u_{\varepsilon, \tau}, v_{\varepsilon, \tau}\right)$ tends to $\left(u_{\varepsilon, 0}, v_{\varepsilon, 0}\right)$ in $C_{N}^{2+d} \times C_{N}^{2+d}$ as $\tau \rightarrow 0$. Thus $\left\|u_{\varepsilon, \tau}\right\|_{L^{\infty}}$ and $\left\|v_{\varepsilon, \tau}\right\|_{L^{\infty}}$ are bounded for small $\tau$. Since $\lim _{\tau \rightarrow 0}\left\|v_{\varepsilon, \tau}-\xi_{\varepsilon}\right\|_{L^{\infty}}=0$ and $\xi_{\varepsilon} \neq 0,\left\|v_{\varepsilon, \tau}\right\|_{L^{\infty}}$ is uniformly bounded away from zero for small $\tau$. Thus $\left\|u_{\varepsilon, \tau}^{p} / v_{\varepsilon, \tau}^{q+1}\right\|_{L^{\infty}}$ and $\left\|u_{\varepsilon, \tau}^{r-1} / v_{\varepsilon, \tau}^{s}\right\|_{L^{\infty}}$ are bounded for small $\tau$. This fact will be used below in Sections 3, 4 and 5 . 
Let $v_{\varepsilon, 0}^{(n)}(n \geqslant 1)$ be the $n$-th eigenvalue of the operator $L_{\varepsilon, 0}$ defined by (2.4) below under the Neumann boundary condition repeated according to its multiplicity.

Theorem B Suppose that the condition (A0) holds. Then for any small $\varepsilon>0$ there is $\tau_{*}=\tau_{*}(\varepsilon)>0$ such that a boundary spike layer $\left(u_{\varepsilon, \tau}(x), v_{\varepsilon, \tau}(x)\right)$ for the full system (1.4) is stable for all $0<\tau<\tau_{*}$, if the three conditions

$$
\text { (A1) } \sigma v_{\varepsilon, 0}^{(1)}<1+s, \quad\left(\text { A2) } \sigma<\frac{(s+1)^{2} \int_{\Omega} \hat{u}_{\varepsilon}^{p+1} d x}{(p+1) q \int_{\Omega} \hat{u}_{\varepsilon}^{2 p} d x} \text { and (A3) } \sigma<\frac{(p+1) q \kappa \int_{\Omega} \hat{u}_{\varepsilon}^{2} d x}{(p-1)^{2} \int_{\Omega} \hat{u}_{\varepsilon}^{p+1} d x}\right.
$$

hold. Here $\hat{u}_{\varepsilon}$ is a solution for (2.1) and $\kappa=\min \left\{\left(\frac{v_{\varepsilon, 0}^{(1)}}{v_{\varepsilon, 0}^{(2)}-v_{\varepsilon, 0}^{(1)}}\right)^{2},\left(\frac{v_{\varepsilon, 0}^{(2)}}{v_{\varepsilon, 0}^{(2)}-v_{\varepsilon, 0}^{(1)}}\right)^{2}\right\}$.

Moreover, in the case $N=1$, (A2) and (A3) hold if the following (A2)' and (A3)'

$$
(\mathrm{A} 2)^{\prime} \quad \sigma<\frac{(3 p+1)(s+1)^{2}}{q(p+1)^{3}} \quad \text { and } \quad(\mathrm{A} 3)^{\prime} \quad \sigma<\frac{2 q \kappa}{(p-1)^{2}}
$$

respectively hold.

In particular, the boundary spike layer given by Theorem $\mathrm{A}$ is stable if $\sigma$ is sufficiently small.

In this theorem and Theorem $\mathrm{C}$ below, by the term "stability" we mean that there is $\alpha>0$ such that the set $\Lambda_{\alpha}$ defined by (1.6) is in the resolvent set of the corresponding linearized operator.

For the case $N=1$ a detailed analysis of the stability of a multispike equilibrium solution for the full system was made by Ward \& Wei [19]. In particular, they determined conditions for which a symmetric $k$-spike equilibrium solution for the full system is stable.

For the case $N=1$, the first and the second eigenvalues $v_{\varepsilon, 0}^{(1)}$ and $v_{\varepsilon, 0}^{(2)}$ are given in Proposition 2.8 .

Theorem C Suppose that the conditions (A0), (A1), (A2) and (A3) hold. For small $\varepsilon>0$, if either

(i) $N=1$, or

(ii) $N \geqslant 2$, and $P_{0}$ is a non-degenerate local maximum point of every principal curvature of $\partial \Omega$,

then a boundary spike layer $\left(u_{\varepsilon, 0}(x), v_{\varepsilon, 0}(x)\right)$ for the shadow system $(1.5)$ is stable.

Moreover, for $N=1$, (A2) and (A3) hold if (A2)' and (A3)' hold.

In particular, if $\sigma$ is sufficiently small, then (A1), (A2) and (A3) are satisfied, and the boundary spike layer is stable.

There are some results on the stability of an interior spike for the shadow system. Wei [16] showed the metastability of a single interior spike for the shadow system provided that either $r=2$ and $1<p \leqslant 1+4 / N$ or $r=p+1$ and $1<p<((N+2) /(N-2))_{+}$ 
hold. That is, the real part of every eigenvalue of the associated linearized operator with positive real part is exponentially small. In general, steady states of (1.4) and (1.5) undergo a Hopf bifurcation as $\sigma$ increases. Ward \& Wei [20] analyzed the eigenvalues of the linearized operator at an interior spike for the shadow system and showed that for $r=2$ and $1<p \leqslant 1+4 / N$ eigenvalues cross into the right half-plane of the complex plane as $\sigma$ increases. They also found an upper and a lower bound for the critical value of $\sigma$.

\subsection{The existence of a boundary spike layer}

Provided that $\tau$ is small, we can construct a boundary spike layer for the system (1.4), perturbing a boundary spike layer for the shadow system (1.5).

We decompose the functional space $C_{N}^{2+d}$ in two subspaces $W_{1}$ and $W$,

$$
C_{N}^{2+d}=W_{1} \oplus W
$$

where $W_{1}=\operatorname{span}\langle 1\rangle, W:=C_{N}^{2+d} \cap Z$ and $Z=\left\{u \in C^{d}(\Omega) \mid \int_{\Omega} u(x) d x=0\right\}$. We also decompose the unknown function $v(x)$ as $v(x)=\xi+\zeta(x)$, where $\xi=\int_{\Omega} v(x) d x$ and $\zeta(x) \in W$.

We define a map $\mathscr{F}=\left(\mathscr{F}_{1}, \mathscr{F}_{2}, \mathscr{F}_{3}\right): \mathbb{R} \times \mathbb{R} \times C_{N}^{2+d} \times W_{1} \times W \rightarrow C^{d} \times W_{1} \times Z$ as

$$
\begin{aligned}
& \mathscr{F}_{1}(\varepsilon, \tau, u, \xi, \zeta)=\varepsilon^{2} \Delta u-u+\frac{u^{p}}{(\xi+\zeta)^{q}}, \\
& \mathscr{F}_{2}(\varepsilon, \tau, u, \xi, \zeta)=\int_{\Omega}\left(\xi+\zeta-\frac{u^{r}}{(\xi+\zeta)^{s}}\right) d x \\
& \mathscr{F}_{3}(\varepsilon, \tau, u, \xi, \zeta)=\Delta \zeta-\tau Q\left(\xi+\zeta-\frac{u^{r}}{(\xi+\zeta)^{s}}\right) .
\end{aligned}
$$

Here $Q$ is the projection operator in $C^{d}$ onto $Z$. When $(\varepsilon, \tau, u, \xi, \zeta)$ satisfies $\mathscr{F}(\varepsilon, \tau, u, \xi, \zeta)=$ 0 , then $(u, \xi+\zeta) \in C_{N}^{2+d} \times C_{N}^{2+d}$ is a solution for the full system (1.4). Let $\left(u_{\varepsilon_{0}, 0}, v_{\varepsilon_{0}, 0}\right)$ be a solution for the shadow system $(1.5)$. Then $\mathscr{F}\left(\varepsilon_{0}, 0, u_{\varepsilon_{0}, 0}, v_{\varepsilon_{0}, 0}, 0\right)=0$. The mapping $\mathscr{F}$ is analytic. Therefore if $D_{(u, \xi, \zeta)} \mathscr{F}\left(\varepsilon_{0}, 0, u_{\varepsilon_{0}, 0}, v_{\varepsilon_{0}, 0}, 0\right)$ is invertible, then by the implicit function theorem for small $\tau$ there is a boundary spike layer $\left(u_{\varepsilon_{0}, \tau}, v_{\varepsilon_{0}, \tau}\right)$ for the problem (1.4) which is close to the boundary spike layer $\left(u_{\varepsilon_{0}, 0}, v_{\varepsilon_{0}, 0}\right)$ for the shadow system (1.5) in $C_{N}^{2+d} \times C_{N}^{2+d}$. It is shown in $\S 4$ that the linear operator $D \mathscr{F}$ is invertible provided that the assumptions of Theorem A are satisfied.

\subsection{The stability of a boundary spike layer}

We consider the stability of the boundary spike layer solution, assuming that this solution exists. We linearize (1.4) at $\left(u_{\varepsilon, \tau}, v_{\varepsilon, \tau}\right)$ and consider the eigenvalue problem

$$
\mathscr{L}_{\varepsilon, \tau}\left(\begin{array}{c}
w \\
z
\end{array}\right)=\left(\begin{array}{cc}
L_{\varepsilon, \tau} & -\frac{q u_{\varepsilon, \tau}^{p}}{v_{\varepsilon, \tau}^{q+1}} \\
\frac{r u_{\varepsilon, \tau}^{r-1}}{\sigma v_{\varepsilon, \tau}^{s}} & \frac{M_{\varepsilon, \tau}}{\sigma}
\end{array}\right)\left(\begin{array}{l}
w \\
z
\end{array}\right)=\lambda\left(\begin{array}{c}
w \\
z
\end{array}\right) \text { for }(w, z) \in Y \times Y .
$$


Here

$$
L_{\varepsilon, \tau}:=\varepsilon^{2} \Delta-1+\frac{p u_{\varepsilon, \tau}^{p-1}}{v_{\varepsilon, \tau}^{q}} \quad \text { and } \quad M_{\varepsilon, \tau}:=\frac{1}{\tau} \Delta-1-\frac{s u_{\varepsilon, \tau}^{r}}{v_{\varepsilon, \tau}^{s+1}} .
$$

For the case $\tau \rightarrow 0$, this eigenvalue problem reduces to

$$
\mathscr{L}_{\varepsilon, 0}\left(\begin{array}{c}
w \\
\eta
\end{array}\right)=\left(\begin{array}{cc}
L_{\varepsilon, 0} & -\frac{q u_{\varepsilon, 0}^{p}}{\xi_{\varepsilon}^{q+1}} \\
\frac{r\left\langle u_{\varepsilon, 0}^{r-1}, \cdot\right\rangle}{\sigma \xi_{\varepsilon}^{s}} & -\frac{1+s}{\sigma}
\end{array}\right)\left(\begin{array}{l}
w \\
\eta
\end{array}\right)=\lambda\left(\begin{array}{l}
w \\
\eta
\end{array}\right) \text { for }(w, \eta) \in Y \times \mathbb{R} .
$$

Here

$$
L_{\varepsilon, 0}:=\varepsilon^{2} \Delta-1+\frac{p u_{\varepsilon, 0}^{p-1}}{\xi_{\varepsilon}^{q}} .
$$

We show that there is $\alpha>0$ such that $\left(\mathscr{L}_{\varepsilon, \tau}-\lambda\right)$ is invertible for all $\lambda \in \Lambda_{\alpha}$. Let $(f, g) \in X \times X$ and consider the problem

$$
\left(\mathscr{L}_{\varepsilon, \tau}-\lambda\right)\left(\begin{array}{c}
w \\
z
\end{array}\right)=\left(\begin{array}{l}
f \\
g
\end{array}\right) .
$$

This system is equivalent to the system

$$
\left(L_{\varepsilon, \tau}-\lambda\right) w-\frac{q u_{\varepsilon, \tau}^{p}}{v_{\varepsilon, \tau}^{q+1}} z=f, \quad\left(\lambda-\frac{M_{\varepsilon, \tau}}{\sigma}\right) z-\frac{1}{\sigma} \frac{r u_{\varepsilon, \tau}^{r-1}}{v_{\varepsilon, \tau}^{s}} w=-g .
$$

The operator $\left(\lambda-M_{\varepsilon, \tau} / \sigma\right)$ is invertible for $\lambda \in \Lambda_{\alpha}$ provided that $\alpha$ is small, and therefore

$$
z=-\left(\lambda-\frac{M_{\varepsilon, \tau}}{\sigma}\right)^{-1}[g]+\left(\lambda-\frac{M_{\varepsilon, \tau}}{\sigma}\right)^{-1}\left[\frac{1}{\sigma} \frac{r u_{\varepsilon, \tau}^{r-1}}{v_{\varepsilon, \tau}^{s}} w\right] .
$$

Substituting (2.7) in the first equation of (2.6) gives

$$
\left(A_{\varepsilon, \tau, \lambda}-\lambda\right) w=F(f, g)
$$

where

$$
\left(A_{\varepsilon, \tau, \lambda}-\lambda\right) w:=\left(L_{\varepsilon, \tau}-\lambda\right) w-\frac{q u_{\varepsilon, \tau}^{p}}{v_{\varepsilon, \tau}^{q+1}}\left(\lambda-\frac{M_{\varepsilon, \tau}}{\sigma}\right)^{-1}\left[\frac{1}{\sigma} \frac{r u_{\varepsilon, \tau}^{r-1}}{v_{\varepsilon, \tau}^{s}} w\right]
$$

and

$$
F(f, g):=f-\frac{q u_{\varepsilon, \tau}^{p}}{v_{\varepsilon, \tau}^{q+1}}\left(\lambda-\frac{M_{\varepsilon, \tau}}{\sigma}\right)^{-1}[g] .
$$

It is noteworthy that $\lambda$ appears in $A_{\varepsilon, \tau, \lambda}$. If $\left(A_{\varepsilon, \tau, \lambda}-\lambda\right)$ is invertible, then (2.8) can be solved with respect to $w$, and $z$ can be found then by a backward substitution. Therefore $\left(\mathscr{L}_{\varepsilon, \tau}-\lambda\right)(\in \mathscr{L}(Y \times Y, X \times X))$ is invertible.

We have to show now that $\left(A_{\varepsilon, \tau, \lambda}-\lambda\right)$ is invertible. Let $\mu_{\varepsilon, \tau}^{(n)}(n \geqslant 1)$ be the $n$-th eigenvalue of $M_{\varepsilon, \tau} / \sigma$ defined by (2.3) under the Neumann boundary condition, repeated according to its multiplicity, and let $\psi_{\varepsilon, \tau}^{(n)}$ be the eigenfunction corresponding to $\mu_{\varepsilon, \tau}^{(n)}$ and satisfying 
$\left\|\psi_{\varepsilon, \tau}^{(n)}\right\|_{X}=1$. From the eigenfunction expansion of $\left(\lambda-M_{\varepsilon, \tau} / \sigma\right)^{-1}$, we have

$$
\left(\lambda-M_{\varepsilon, \tau} / \sigma\right)^{-1}=\sum_{n \geqslant 1} \frac{\left\langle\cdot, \psi_{\varepsilon, \tau}^{(n)}\right\rangle}{\lambda-\mu_{\varepsilon, \tau}^{(n)}} \psi_{\varepsilon, \tau}^{(n)} .
$$

We decompose the operator $\left(\lambda-M_{\varepsilon, \tau} / \sigma\right)^{-1}$ in two parts,

$$
\left(\lambda-\frac{M_{\varepsilon, \tau}}{\sigma}\right)^{-1}[\cdot]=\frac{\left\langle\cdot, \psi_{\varepsilon, \tau}^{(1)}\right\rangle}{\lambda-\mu_{\varepsilon, \tau}^{(1)}} \psi_{\varepsilon, \tau}^{(1)}+\left(\lambda-\frac{M_{\varepsilon, \tau}}{\sigma}\right)^{\dagger}[\cdot] .
$$

Here

$$
\left(\lambda-\frac{M_{\varepsilon, \tau}}{\sigma}\right)^{\dagger}[\cdot]:=\sum_{n \geqslant 2} \frac{\left\langle\cdot, \psi_{\varepsilon, \tau}^{(n)}\right\rangle}{\lambda-\mu_{\varepsilon, \tau}^{(n)}} \psi_{\varepsilon, \tau}^{(n)}: X \rightarrow X \cap \operatorname{span}\left\langle\psi_{\varepsilon, \tau}^{(1)}\right\rangle^{\perp} .
$$

We will prove that $\mu_{\varepsilon, \tau}^{(n)}$ satisfies

$$
\lim _{\tau \rightarrow 0} \sigma \mu_{\varepsilon, \tau}^{(1)}=-1-s, \quad \lim _{\tau \rightarrow 0} \mu_{\varepsilon, \tau}^{(n)}=-\infty \text { for } n \geqslant 2 .
$$

On the other hand, from (2.12) it follows that

$$
\left\|\left(\lambda-\frac{M_{\varepsilon, \tau}}{\sigma}\right)^{\dagger}\right\|_{\mathscr{L}(X, X)} \leqslant \frac{1}{\left|\operatorname{Re}(\lambda)-\mu_{\varepsilon, \tau}^{(2)}\right|} \quad \text { for } \operatorname{Re}(\lambda)>\mu_{\varepsilon, \tau}^{(2)} .
$$

Here $\|\cdot\|_{\mathscr{L}(X, X)}$ denotes the operator norm. From (2.14) and (2.13), it is easy to see that

$$
\lim _{\tau \rightarrow 0}\left\|\left(\lambda-\frac{M_{\varepsilon, \tau}}{\sigma}\right)^{\dagger}\right\|_{\mathscr{L}(X, X)}=0 \text { uniformly for all } \lambda \in \Lambda_{\alpha} .
$$

Moreover, we can see by (2.11) that $\left(\lambda-M_{\varepsilon, \tau} / \sigma\right)$ is invertible for $\lambda \in \Lambda_{\alpha}$ provided that $\tau>0$ is sufficiently small.

We decompose $A_{\varepsilon, \tau, \lambda}$ as

$$
A_{\varepsilon, \tau, \lambda}=L_{\varepsilon, \tau}+B_{\varepsilon, \tau, \lambda}+R_{\varepsilon, \tau, \lambda}
$$

Here

$$
B_{\varepsilon, \tau, \lambda} w:=-\frac{q u_{\varepsilon, \tau}^{p}}{\left(\lambda-\mu_{\varepsilon, \tau}^{(1)}\right) v_{\varepsilon, \tau}^{q+1}}\left\langle\frac{1}{\sigma} \frac{r u_{\varepsilon, \tau}^{r-1}}{v_{\varepsilon, \tau}^{s}} w, \psi_{\varepsilon, \tau}^{(1)}\right\rangle \psi_{\varepsilon, \tau}^{(1)}
$$

and

$$
R_{\varepsilon, \tau, \lambda} w:=-\frac{q u_{\varepsilon, \tau}^{p}}{v_{\varepsilon, \tau}^{q+1}}\left(\lambda-\frac{M_{\varepsilon, \tau}}{\sigma}\right)^{\dagger}\left[\frac{1}{\sigma} \frac{r u_{\varepsilon, \tau}^{r-1}}{v_{\varepsilon, \tau}^{s}} w\right] .
$$

Since for small $\tau>0\left\|u_{\varepsilon, \tau}^{p} / v_{\varepsilon, \tau}^{q+1}\right\|_{L^{\infty}}$ and $\left\|u_{\varepsilon, \tau}^{r-1} / v_{\varepsilon, \tau}^{s}\right\|_{L^{\infty}}$ are bounded, it is easy to see by (2.15) that

$$
\left\|R_{\varepsilon, \tau, \lambda}\right\|_{\mathscr{L}(X, X)} \rightarrow 0 \text { as } \tau \rightarrow 0 .
$$

(For the details, see the proof of Lemma 5.2.) 
Let $(f, l) \in X \times \mathbb{R}$. To study the stability of the boundary spike layer for the shadow system (1.5), we will consider the problem

$$
\left(\mathscr{L}_{\varepsilon, 0}-\lambda\right)\left(\begin{array}{l}
w \\
\eta
\end{array}\right)=\left(\begin{array}{l}
f \\
l
\end{array}\right) .
$$

This system is equivalent to

$$
\left(L_{\varepsilon, 0}-\lambda\right) w-\frac{q u_{\varepsilon, 0}^{p}}{\xi_{\varepsilon}^{q+1}} \eta=f, \quad \frac{r}{\xi_{\varepsilon}}\left\langle u_{\varepsilon, 0}^{r-1}, w\right\rangle-(\sigma \lambda+1+s) \eta=l .
$$

Solving the second equation of (2.19) with respect to $\eta$, we obtain

$$
\eta=\frac{1}{\sigma \lambda+1+s}\left\langle\frac{r u_{\varepsilon, 0}^{r-1}}{\xi_{\varepsilon}^{s}} w, 1\right\rangle-\frac{l}{\sigma \lambda+1+s}
$$

Here we use the equality $\frac{r}{\xi_{\varepsilon}^{s}}\left\langle u_{\varepsilon, 0}^{r-1}, w\right\rangle=\left\langle\frac{r u_{\varepsilon, 0}^{r-1}}{\xi_{\varepsilon}^{s}} w, 1\right\rangle$. Substituting (2.20) into the first equation of (2.19), we obtain

$$
\left(A_{\varepsilon, 0, \lambda}-\lambda\right) w=\tilde{F}(f, l)
$$

Here

$$
\begin{aligned}
A_{\varepsilon, 0, \lambda} & :=L_{\varepsilon, 0}+B_{\varepsilon, 0, \lambda} \\
B_{\varepsilon, 0, \lambda} w & :=-\frac{q u_{\varepsilon, 0}^{p}}{(\sigma \lambda+1+s) \xi_{\varepsilon}^{q+1}}\left\langle\frac{r u_{\varepsilon, 0}^{r-1}}{\xi_{\varepsilon}^{s}} w, 1\right\rangle
\end{aligned}
$$

and

$$
\tilde{F}(f, l):=f-\frac{q l u_{\varepsilon, 0}^{p}}{(\sigma \lambda+1+s) \xi_{\varepsilon}^{q+1}} .
$$

Notice that the eigenvalue $\lambda$ appears in $A_{\varepsilon, 0, \lambda}$.

We study the invertibility of $\left(A_{\varepsilon, 0, \lambda}-\lambda\right)$ by using the Sherman-Morrison formula. If $\left(A_{\varepsilon, 0, \lambda}-\lambda\right)$ is invertible for $\lambda \in \Lambda_{\alpha} \backslash \mathbb{R}$ (see Lemma 4.4), we consider the case that $\lambda \in \mathbb{R}$. Since

$$
B_{\varepsilon, 0, \lambda} w=\beta\langle w, \Phi\rangle \Phi,
$$

where $\beta=-q r /\left\{(\sigma \lambda+1+s) \xi_{\varepsilon}^{q+s+1}\right\}$ and $\Phi=u_{\varepsilon, 0}^{p}$, the rank of the operator $B_{\varepsilon, 0, \lambda}$ is one. It is important to note that the assumption (A0) guarantees that $B_{\varepsilon, 0, \lambda}$ is self-adjoint provided that $\lambda \in \mathbb{R}$. When $\lambda$ is not an eigenvalue of $L_{\varepsilon, 0}$, we can express $\left(A_{\varepsilon, 0, \lambda}-\lambda\right)^{-1}$ by the Sherman-Morrison formula as

$$
\begin{aligned}
\left(A_{\varepsilon, 0, \lambda}-\lambda\right)^{-1} & =\left(I+\left(L_{\varepsilon, 0}-\lambda\right)^{-1} B_{\varepsilon, 0, \lambda}\right)^{-1}\left(L_{\varepsilon, 0}-\lambda\right)^{-1} \\
& =\left(I-\frac{\left(L_{\varepsilon, 0}-\lambda\right)^{-1} B_{\varepsilon, 0, \lambda}}{h(\lambda)}\right)\left(L_{\varepsilon, 0}-\lambda\right)^{-1},
\end{aligned}
$$

where

$$
h(\lambda)=1+\beta\left\langle\left(L_{\varepsilon, 0}-\lambda\right)^{-1} \Phi, \Phi\right\rangle .
$$


If $h(\lambda) \neq 0$, then $\left(A_{\varepsilon, 0, \lambda}-\lambda\right)$ is invertible. If $h(\lambda)=0$, then $\left(L_{\varepsilon, 0}-\lambda\right)^{-1} \Phi$ is in the kernel of $\left(A_{\varepsilon, 0, \lambda}-\lambda\right)$. Therefore $\left(A_{\varepsilon, 0, \lambda}-\lambda\right)$ is invertible if and only if $h(\lambda) \neq 0$ provided that $\lambda$ is not an eigenvalue of $L_{\varepsilon, 0}$. Detailed analysis will be done in Lemmas 4.1, 4.2 and 4.3.

We now study the eigenvalues of $L_{\varepsilon, 0}$. Assume that $U(x)$ is a boundary spike layer for the system

$$
\Delta U-U+U^{p}=0 \text { in } \mathbb{R}_{+}^{N}, \quad \frac{\partial U}{\partial x_{N}}=0 \text { on } \partial \mathbb{R}_{+}^{N}, \quad U>0 \text { and } U \in H^{1}\left(\mathbb{R}_{+}^{N}\right),
$$

and $U^{\prime}(|x|)$ denotes the radial derivative of $U$ with respect to $|x|$.

Proposition 2.4 For $N \geqslant 2$, and $\mathbb{R}_{+}^{N}=\left\{x=\left(x_{1}, x_{2}, \cdots, x_{N}\right) \in \mathbb{R}^{N} \mid x_{N}>0\right\}$, The eigenvalue problem

$$
\Delta \phi-\phi+p U^{p-1} \phi=\rho \phi \text { in } \mathbb{R}_{+}^{N}, \quad \frac{\partial \phi}{\partial x_{N}}=0 \text { on } \partial \mathbb{R}_{+}^{N} \text { and } \phi \in H^{1}\left(\mathbb{R}_{+}^{N}\right),
$$

admits the set of eigenvalues

$$
\rho_{1}>0, \rho_{2}=\cdots=\rho_{N}=0, \rho_{N+1}<0, \cdots .
$$

Proposition 2.5 [17] Let $\hat{u}_{\varepsilon}$ be a boundary spike layer for (2.1) for $N \geqslant 2$. Then for $\varepsilon$ sufficiently small, the eigenvalue problem

$$
\varepsilon^{2} \Delta \phi_{\varepsilon}-\phi_{\varepsilon}+p \hat{u}_{\varepsilon}^{p-1} \phi_{\varepsilon}=\theta_{\varepsilon} \phi_{\varepsilon} \text { in } \Omega, \quad \frac{\partial \phi_{\varepsilon}}{\partial n}=0 \quad \text { on } \partial \Omega
$$

admits exactly $(N-1)$ eigenvalues $\theta_{\varepsilon}^{(1)} \leqslant \theta_{\varepsilon}^{(2)} \leqslant \cdots \leqslant \theta_{\varepsilon}^{(N-1)}$ in the interval $\left[\frac{1}{2} \rho_{N+1}, \frac{1}{2} \rho_{1}\right]$, where $\rho_{1}$ and $\rho_{N+1}$ are given by Proposition 2.4.

Moreover,

$$
\frac{\theta_{\varepsilon}^{(j)}}{\varepsilon^{2}} \rightarrow \eta_{0} \lambda_{j}, \quad j=1,2, \cdots, N-1,
$$

where $\lambda_{1} \leqslant \lambda_{2} \leqslant \cdots \leqslant \lambda_{N-1}$ are the eigenvalues of the matrix $G\left(P_{0}\right)$ defined by Definition 2.2 , and

$$
\eta_{0}=\frac{N-1}{N+1}\left(\int_{\mathbb{R}_{+}^{N}}\left(U^{\prime}(|x|)\right)^{2} x_{N} d x\right) /\left(\int_{\mathbb{R}_{+}^{N}}\left(\frac{\partial U}{\partial x_{1}}\right)^{2} d x\right)>0
$$

Remark 2.6 We remark that if every principal curvature has a non-degenerate local maximum at $P_{0} \in \partial \Omega$, then all eigenvalues of the matrix $G\left(P_{0}\right)$ defined in Definition 2.2 are negative.

Using a scaling argument and a diagonal argument, we can see that each eigenvalue of (2.27) tends to an eigenvalue of (2.26) as $\varepsilon \rightarrow 0$. Thus if $\lambda_{j}$ is negative for all $j \in\{1,2, \cdots, N-1\}$, then for small $\varepsilon>0$ only the first eigenvalue of (2.27) is positive. Suppose that the contrary is true. Then there is a positive eigenvalue that is not principal for small $\varepsilon>0$. That eigenvalue should tend to either $\rho_{1}$, or 0 as $\varepsilon \rightarrow 0$. This is a contradiction. 
Corollary 2.7 For $N \geqslant 2$, suppose that all the assumptions of Remark 2.6 hold. Then for small $\varepsilon>0$ only the first eigenvalue of (2.27) is positive, and all other eigenvalues are negative, that is, $v_{\varepsilon, 0}^{(1)}>0$ and $v_{\varepsilon, 0}^{(n)}<0$ for $n \geqslant 2$. In particular, for small $\varepsilon>0$ zero is not an eigenvalue. Therefore, $L_{\varepsilon, 0}$ is invertible under the Neumann boundary condition.

In the case $N=1$, the spectrum of $L_{\varepsilon, 0}$ consists of the odd-numbered eigenvalues of the linearized operator at an interior one-spike equilibrium. Therefore, we easily obtain the following proposition [1].

Proposition 2.8 [1] For $N=1$, for $p>1$ and for $\varepsilon \rightarrow 0$, the two largest eigenvalues of a linearized operator at a boundary spike layer $L_{\varepsilon, 0}$, satisfy the asymptotic formulae

$$
v_{\varepsilon, 0}^{(1)}=\frac{1}{4}(p-1)(p+3)+O\left(\exp \frac{-2}{\varepsilon}\right)
$$

and

$$
v_{\varepsilon, 0}^{(2)}= \begin{cases}\frac{1}{4}(p-1)(p-5)+O\left(\exp \frac{-2}{\varepsilon}\right), & \text { if } p<3 \\ <-\frac{1}{2}, & \text { if } p \geqslant 3\end{cases}
$$

Therefore for $N=1$ the same conclusion as Corollary 2.7 holds.

We now return to the analysis of $A_{\varepsilon, \tau, \lambda}$. We show that $\left(A_{\varepsilon, \tau, \lambda}-\lambda\right)$ is close to $\left(A_{\varepsilon, 0, \lambda}-\lambda\right)$. We have

$$
A_{\varepsilon, \tau, \lambda}-A_{\varepsilon, 0, \lambda}=\left(L_{\varepsilon, \tau}-L_{\varepsilon, 0}\right)+\left(B_{\varepsilon, \tau, \lambda}-B_{\varepsilon, 0, \lambda}\right)+R_{\varepsilon, \tau, \lambda}
$$

Here

$$
\begin{gathered}
\left(L_{\varepsilon, \tau}-L_{\varepsilon, 0}\right) w=p\left(\frac{u_{\varepsilon, \tau}^{p-1}}{v_{\varepsilon, \tau}^{q}}-\frac{u_{\varepsilon, 0}^{p-1}}{\xi_{\varepsilon}^{q}}\right) w, \\
\left(B_{\varepsilon, \tau, \lambda}-B_{\varepsilon, 0, \lambda}\right) w=-\frac{q u_{\varepsilon, \tau}^{p}}{\left(\lambda-\mu_{\varepsilon, \tau}^{(1)}\right) v_{\varepsilon, \tau}^{q+1}}\left\langle\frac{1}{\sigma} \frac{r u_{\varepsilon, \tau}^{r-1}}{v_{\varepsilon, \tau}^{s}} w, \psi_{\varepsilon, \tau}^{(1)}\right\rangle \psi_{\varepsilon, \tau}^{(1)} \\
+\frac{q u_{\varepsilon, 0}^{p}}{(\sigma \lambda+1+s) \xi_{\varepsilon}^{q+1}}\left\langle\frac{r u_{\varepsilon, 0}^{r-1}}{\xi_{\varepsilon}^{s}} w, 1\right\rangle,
\end{gathered}
$$

and $R_{\varepsilon, \tau, \lambda}$ is defined by (2.16). We see by Theorem A and Lemma 3.3 below that $\lim _{\tau \rightarrow 0}\left\|u_{\varepsilon, \tau}-u_{\varepsilon, 0}\right\|_{C^{0}}=0, \lim _{\tau \rightarrow 0}\left\|v_{\varepsilon, \tau}-\xi_{\varepsilon}\right\|_{C^{0}}=0, \lim _{\tau \rightarrow 0}\left\|\left(\psi_{\varepsilon, \tau}^{(1)}\right)^{2}-1\right\|_{X}=0$ and $\lim _{\tau \rightarrow 0} \sigma \mu_{\varepsilon, \tau}^{(1)}=-1-s$. Therefore, $\left(L_{\varepsilon, \tau}-L_{\varepsilon, 0}\right)$ and $\left(B_{\varepsilon, \tau, \lambda}-B_{\varepsilon, 0, \lambda}\right)$ converge to zero uniformly for $\lambda \in \Lambda_{\alpha}$ in the sense of the operator norm. We can see by (2.17) that $R_{\varepsilon, \tau, \lambda}$ tends to zero uniformly for $\lambda \in \Lambda_{\alpha}$. Thus $\lim _{\tau \rightarrow 0}\left\|A_{\varepsilon, \tau, \lambda}-A_{\varepsilon, 0, \lambda}\right\|_{\mathscr{L}(X, X)}=0$ uniformly for $\lambda \in \Lambda_{\alpha}$ (see Lemma 5.1). The operator $\left(A_{\varepsilon, \tau, \lambda}-\lambda\right)^{-1}$ can be constructed by perturbing $\left(A_{\varepsilon, 0, \lambda}-\lambda\right)^{-1}$. We have

$$
\left(A_{\varepsilon, \tau, \lambda}-\lambda\right)^{-1}=(I+T)^{-1}\left(A_{\varepsilon, 0, \lambda}-\lambda\right)^{-1} \in \mathscr{L}(X, X),
$$

where $T=\left(A_{\varepsilon, 0, \lambda}-\lambda\right)^{-1}\left(A_{\varepsilon, \tau, \lambda}-A_{\varepsilon, 0, \lambda}\right)$, and $I \in \mathscr{L}(X, X)$ is the identity operator. The operator $(I+T)$ is invertible and $(I+T)^{-1}$ can be expressed by a Neumann series (see 
(5.7) below) if

$$
\|T\|_{\mathscr{L}(X, X)}<1 / 2 \text { uniformly for } \lambda \in \Lambda_{\alpha} .
$$

We will prove that this condition holds in Lemma 5.2 below.

\section{Preliminaries}

The equalities

$$
u_{\varepsilon, 0}^{p}=\frac{\xi_{\varepsilon}^{p}}{p-1} L_{\varepsilon, 0}\left[u_{\varepsilon, 0}\right]
$$

and

$$
\xi_{\varepsilon}^{s+1}=\int_{\Omega} u_{\varepsilon, 0}^{r} d x
$$

can be obtained by direct calculations.

Let $\mu_{\varepsilon, \tau}^{(n)}$ (where $n \geqslant 1$ ) be the $n$-th eigenvalue of the operator $M_{\varepsilon, \tau} / \sigma$ defined by (2.3) under the Neumann boundary condition, repeated according to its multiplicity, and let $\psi_{\varepsilon, \tau}^{(n)}$ be the eigenfunction corresponding to $\mu_{\varepsilon, \tau}^{(n)}$.

Lemma 3.1 Let $\varepsilon>0$ be small. Then for each $n \geqslant 2$,

$$
\lim _{\tau \rightarrow 0} \mu_{\varepsilon, \tau}^{(n)}=-\infty \text { uniformly for small } \sigma .
$$

Proof Since $s u_{\varepsilon, \tau}^{r} / v_{\varepsilon, \tau}^{s+1}>0$, and by the comparison theorem of the eigenvalues, $\mu_{\varepsilon, \tau}^{(2)}<\tilde{\mu}_{\varepsilon, \tau}^{(2)}$, where $\tilde{\mu}_{\varepsilon, \tau}^{(2)}$ is the second eigenvalue of the eigenvalue problem

$$
\frac{1}{\tau} \Delta \tilde{\psi}-\tilde{\psi}=\sigma \tilde{\mu} \tilde{\psi} \quad \text { in } \Omega \text { and } \quad \frac{\partial \tilde{\psi}}{\partial n}=0 \quad \text { on } \partial \Omega
$$

Let $\hat{\mu}^{(2)}$ be the second eigenvalue of the Laplace operator under the Neumann boundary condition. Then $\sigma \tilde{\mu}^{(2)}=-1+\hat{\mu}^{(2)} / \tau$. The theory of partial differential equations of the second order yields that the first eigenvalue of the Laplace operator is simple. Since $\hat{\mu}^{(1)}=0$, we have $\hat{\mu}^{(2)}<\hat{\mu}^{(1)}=0$. Therefore

$$
\sigma \tilde{\mu}^{(2)}=-1+\frac{\hat{\mu}^{(2)}}{\tau} \rightarrow-\infty \text { as } \tau \rightarrow 0
$$

The proof is completed.

Lemma 3.2 Let $\varepsilon>0$ be small. Then $\sigma \mu_{\varepsilon, \tau}^{(1)}$ is bounded for small $\tau$.

Proof By Remark 2.3, for small $\tau$ there exists $M>0$ such that $0 \leqslant s u_{\varepsilon, \tau}^{r} / v_{\varepsilon, \tau}^{s+1} \leqslant M$. It follows from the comparison principle of eigenvalues that $\underline{\mu}^{(1)} \leqslant \sigma \mu_{\varepsilon, \tau}^{(1)} \leqslant \bar{\mu}^{(1)}$, where $\underline{\mu}^{(1)}$ and $\bar{\mu}^{(1)}$ are respectively the first eigenvalues of the eigenvalue problem

$$
\frac{1}{\tau} \Delta \underline{\phi}-\underline{\phi}-M \underline{\phi}=\underline{\mu \phi} \text { in } \Omega \text { and } \frac{\partial \underline{\phi}}{\partial n}=0 \text { on } \partial \Omega,
$$


and

$$
\frac{1}{\tau} \Delta \bar{\phi}-\bar{\phi}=\bar{\mu} \bar{\phi} \text { in } \Omega \text { and } \frac{\partial \bar{\phi}}{\partial n}=0 \text { on } \partial \Omega \text {. }
$$

Since $\underline{\mu}^{(1)}=-1-M$ and $\bar{\mu}^{(1)}=-1$, we obtain the desired conclusion.

Lemma 3.3 For $\varepsilon>0$ be small,

(i) $\lim _{\tau \rightarrow 0}\left\|\left(\psi_{\varepsilon, \tau}^{(1)}\right)^{2}-1\right\|_{X}=0$ and

(ii) $\lim _{\tau \rightarrow 0} \sigma \mu_{\varepsilon, \tau}^{(1)}=-1-s$

hold.

Proof Let $(\operatorname{span}\langle 1\rangle)^{\perp}$ be the orthogonal complement space of the space span $\langle 1\rangle$ in $L^{2}(\Omega)$. We decompose the first eigenfunction as

$$
\psi_{\varepsilon, \tau}^{(1)}:=\bar{\psi}_{\varepsilon, \tau}+\psi_{\varepsilon, \tau}^{\perp} \in(\operatorname{span}\langle 1\rangle) \oplus(\operatorname{span}\langle 1\rangle)^{\perp},
$$

where $\bar{\psi}_{\varepsilon, \tau}=\int_{\Omega} \psi_{\varepsilon, \tau}^{(1)} d x$ and $\psi_{\varepsilon, \tau}^{\perp}=\psi_{\varepsilon, \tau}^{(1)}-\bar{\psi}_{\varepsilon, \tau}$. In particular, $\int_{\Omega} \psi_{\varepsilon, \tau}^{\perp} d x=0$. Since $\psi_{\varepsilon, \tau}^{(1)}$ is the eigenfunction corresponding to $\mu_{\varepsilon, \tau}^{(1)}$, we have

$$
\frac{1}{\tau} \Delta \psi_{\varepsilon, \tau}^{(1)}-\psi_{\varepsilon, \tau}^{(1)}-s \frac{u_{\varepsilon, \tau}^{r}}{v_{\varepsilon, \tau}^{s+1}} \psi_{\varepsilon, \tau}^{(1)}=\sigma \mu_{\varepsilon, \tau}^{(1)} \psi_{\varepsilon, \tau}^{(1)} .
$$

Therefore, $\bar{\psi}_{\varepsilon, \tau}$ and $\psi_{\varepsilon, \tau}^{\perp}$ satisfy

$$
\frac{1}{\tau} \Delta \psi_{\varepsilon, \tau}^{\perp}-\left(\bar{\psi}_{\varepsilon, \tau}+\psi_{\varepsilon, \tau}^{\perp}\right)-s \frac{u_{\varepsilon, \tau}^{r}}{v_{\varepsilon, \tau}^{s+1}}\left(\bar{\psi}_{\varepsilon, \tau}+\psi_{\varepsilon, \tau}^{\perp}\right)=\sigma \mu_{\varepsilon, \tau}^{(1)}\left(\bar{\psi}_{\varepsilon, \tau}+\psi_{\varepsilon, \tau}^{\perp}\right) .
$$

Integrating both sides of (3.5), and using the equality $\int_{\Omega} \Delta \psi_{\varepsilon, \tau}^{\perp} d x=0$, we obtain

$$
\bar{\psi}_{\varepsilon, \tau}\left(1+\sigma \mu_{\varepsilon, \tau}^{(1)}+s \int_{\Omega} \frac{u_{\varepsilon, \tau}^{r}}{v_{\varepsilon, \tau}^{s+1}} d x\right)=-s \int_{\Omega} \frac{u_{\varepsilon, \tau}^{r}}{v_{\varepsilon, \tau}^{s+1}} \psi_{\varepsilon, \tau}^{\perp} d x
$$

By contradiction, we will show that

$$
1+\sigma \mu_{\varepsilon, \tau}^{(1)}+s \int_{\Omega} \frac{u_{\varepsilon, \tau}^{r}}{v_{\varepsilon, \tau}^{s+1}} d x \neq 0
$$

Suppose that the equality holds. If $\bar{\psi}_{\varepsilon, \tau}+\psi_{\varepsilon, \tau}^{\perp}$ is an eigenfunction, then $c \bar{\psi}_{\varepsilon, \tau}+\psi_{\varepsilon, \tau}^{\perp}(c \in \mathbb{R})$ is also an eigenfunction. Since the first eigenvalue $\mu_{\varepsilon, \tau}^{(1)}$ is simple, the dimension of the eigenspace corresponding to $\mu_{\varepsilon, \tau}^{(1)}$ is one. Therefore, $\bar{\psi}_{\varepsilon, \tau}+\psi_{\varepsilon, \tau}^{\perp}$ and $c \bar{\psi}_{\varepsilon, \tau}+\psi_{\varepsilon, \tau}^{\perp}$ should be parallel for all $c \in \mathbb{R}$. Hence $\psi_{\varepsilon, \tau}^{\perp}=0$ and $\bar{\psi}_{\varepsilon, \tau}=0$, and hence (3.7) holds.

By (3.6), we have

$$
\bar{\psi}_{\varepsilon, \tau}=-s \int_{\Omega} \frac{u_{\varepsilon, \tau}^{r}}{v_{\varepsilon, \tau}^{s+1}} \psi_{\varepsilon, \tau}^{\perp} d x /\left(1+\sigma \mu_{\varepsilon, \tau}^{(1)}+s \int_{\Omega} \frac{u_{\varepsilon, \tau}^{r}}{v_{\varepsilon, \tau}^{s+1}} d x\right) .
$$


On the other hand, by (3.5) we have

$$
\frac{1}{\tau} \Delta \psi_{\varepsilon, \tau}^{\perp}=\left(1+\sigma \mu_{\varepsilon, \tau}^{(1)}+s \frac{u_{\varepsilon, \tau}^{r}}{v_{\varepsilon, \tau}^{s+1}}\right)\left(\bar{\psi}_{\varepsilon, \tau}+\psi_{\varepsilon, \tau}^{\perp}\right) .
$$

Owing to (3.6), we can solve the above equation with respect to $\psi_{\varepsilon, \tau}^{\perp}$ on the left-hand side. We have

$$
\psi_{\varepsilon, \tau}^{\perp}=\tau \Delta^{-1}\left[\left(1+\sigma \mu_{\varepsilon, \tau}^{(1)}+s \frac{u_{\varepsilon, \tau}^{r}}{v_{\varepsilon, \tau}^{s+1}}\right)\left(\bar{\psi}_{\varepsilon, \tau}+\psi_{\varepsilon, \tau}^{\perp}\right)\right],
$$

where $\Delta^{-1}$ denotes the inverse of the operator $\Delta \in \mathscr{L}(Y, X)$ under the Neumann boundary condition. Therefore, we have

$$
\left\|\psi_{\varepsilon, \tau}^{\perp}\right\|_{Y} \leqslant \tau\left\|\Delta^{-1}\right\|_{\mathscr{L}(X, Y)}\left\|1+\sigma \mu_{\varepsilon, \tau}^{(1)}+s \frac{u_{\varepsilon, \tau}^{r}}{v_{\varepsilon, \tau}^{s+1}}\right\|_{L^{\infty}}\left\|\bar{\psi}_{\varepsilon, \tau}+\psi_{\varepsilon, \tau}^{\perp}\right\|_{X} .
$$

By Lemma 3.2, there exists $C>0$ independent of $\tau$ such that

$$
\left\|1+\sigma \mu_{\varepsilon, \tau}^{(1)}+s \frac{u_{\varepsilon, \tau}^{r}}{v_{\varepsilon, \tau}^{s+1}}\right\|_{L^{\infty}} \leqslant C \text { for small } \tau .
$$

Here $\left\|\Delta^{-1}\right\|_{\mathscr{L}(X, Y)}$ is bounded, and $\left\|\bar{\psi}_{\varepsilon, \tau}+\psi_{\varepsilon, \tau}^{\perp}\right\|_{X}=1$. Hence, by (3.8),

$$
\left\|\psi_{\varepsilon, \tau}^{\perp}\right\|_{Y} \leqslant C \tau \text {. }
$$

Therefore for $\tau \rightarrow 0$ we have the limits

$$
\int_{\Omega}\left(\psi_{\varepsilon, \tau}^{\perp}\right)^{2} d x \leqslant C \tau^{2} \rightarrow 0 \text { as } \tau \rightarrow 0,
$$

and

$$
\frac{1}{\tau} \int_{\Omega}\left(\nabla \psi_{\varepsilon, \tau}^{\perp}\right)^{2} d x \leqslant C \tau \rightarrow 0 \text { as } \tau \rightarrow 0 .
$$

Since $1=\bar{\psi}_{\varepsilon, \tau}^{2}+\int_{\Omega}\left(\psi_{\varepsilon, \tau}^{\perp}\right)^{2} d x$, we see that as $\tau \rightarrow 0$

$$
\bar{\psi}_{\varepsilon, \tau}^{2}=1-\int_{\Omega}\left(\psi_{\varepsilon, \tau}^{\perp}\right)^{2} d x \rightarrow 1
$$

Thus the proof of (i) is completed.

We will show that

$$
\int_{\Omega} \frac{u_{\varepsilon, \tau}^{r}}{v_{\varepsilon, \tau}^{s+1}}\left(\psi_{\varepsilon, \tau}^{(1)}\right)^{2} d x \rightarrow 1 \text { as } \tau \rightarrow 0
$$

We have

$$
\begin{aligned}
\int_{\Omega} \frac{u_{\varepsilon, \tau}^{r}}{v_{\varepsilon, \tau}^{s+1}}\left(\psi_{\varepsilon, \tau}^{(1)}\right)^{2} d x-1 & =\int_{\Omega} \frac{u_{\varepsilon, \tau}^{r}}{v_{\varepsilon, \tau}^{s+1}}\left(\psi_{\varepsilon, \tau}^{(1)}\right)^{2} d x-\int_{\Omega} \frac{u_{\varepsilon, 0}^{r}}{\xi_{\varepsilon}^{s+1}} d x \\
& =\int_{\Omega} \frac{u_{\varepsilon, \tau}^{r}}{v_{\varepsilon, \tau}^{s+1}}\left\{\left(\psi_{\varepsilon, \tau}^{(1)}\right)^{2}-1\right\} d x+\int_{\Omega} u_{\varepsilon, \tau}^{r}\left(\frac{1}{v_{\varepsilon, \tau}^{s+1}}-\frac{1}{\xi_{\varepsilon}^{s+1}}\right) d x \\
& =I_{1}+I_{2},
\end{aligned}
$$


where we use (3.3). By Remark 2.3, we obtain

$$
I_{2} \rightarrow 0 \text { as } \tau \rightarrow 0
$$

Furthermore, we have

$$
\left|I_{1}\right| \leqslant \int_{\Omega}\left|\frac{u_{\varepsilon, \tau}^{r}}{v_{\varepsilon, \tau}^{s+1}}\left\{\left(\psi_{\varepsilon, \tau}^{(1)}\right)^{2}-1\right\}\right| d x \leqslant\left\|\frac{u_{\varepsilon, \tau}^{r}}{v_{\varepsilon, \tau}^{s+1}}\right\|_{L^{\infty}} \int_{\Omega}\left|\left(\psi_{\varepsilon, \tau}^{(1)}\right)^{2}-1\right| d x
$$

and then

$$
\begin{aligned}
\int_{\Omega}\left|\left(\psi_{\varepsilon, \tau}^{(1)}\right)^{2}-1\right| d x & =\int_{\Omega}\left|\bar{\psi}_{\varepsilon, \tau}^{2}+2 \bar{\psi}_{\varepsilon, \tau} \psi_{\varepsilon, \tau}^{\perp}+\left(\psi_{\varepsilon, \tau}^{\perp}\right)^{2}-1\right| d x \\
& \leqslant|\Omega|\left|\bar{\psi}_{\varepsilon, \tau}^{2}-1\right|+2\left|\bar{\psi}_{\varepsilon, \tau}\right| \int_{\Omega}\left|\psi_{\varepsilon, \tau}^{\perp}\right| d x+\int_{\Omega}\left(\psi_{\varepsilon, \tau}^{\perp}\right)^{2} d x \\
& \leqslant|\Omega|\left|\bar{\psi}_{\varepsilon, \tau}^{2}-1\right|+2\left|\bar{\psi}_{\varepsilon, \tau}\right|\left(|\Omega| \int_{\Omega}\left(\psi_{\varepsilon, \tau}^{\perp}\right)^{2} d x\right)^{1 / 2}+\int_{\Omega}\left(\psi_{\varepsilon, \tau}^{\perp}\right)^{2} d x
\end{aligned}
$$

where we use Hölder's inequality. By (3.9) and (3.11), the left-hand side of (3.15) tends to zero as $\tau \rightarrow 0$. Thus $I_{1} \rightarrow 0$ as $\tau \rightarrow 0$ as well, and hence by (3.14) and (3.15) the equation (3.12) holds. Multiplying the both sides of (3.4) by $\psi_{\varepsilon, \tau}^{(1)}$ and integrating, we have

$$
\sigma \mu_{\varepsilon, \tau}^{(1)}=-1-s \int_{\Omega} \frac{u_{\varepsilon, \tau}^{r}}{v_{\varepsilon, \tau}^{s+1}}\left(\psi_{\varepsilon, \tau}^{(1)}\right)^{2} d x-\frac{1}{\tau} \int_{\Omega}\left|\nabla \psi_{\varepsilon, \tau}^{(1)}\right|^{2} d x
$$

We can see by (3.10) and (3.12), that $\sigma \mu_{\varepsilon, \tau}^{(1)} \rightarrow-1-s$ as $\tau \rightarrow 0$. The proof of (ii) is completed.

Remark 3.4 The theory of partial differential equations of the second order shows that the first eigenfunction $\psi_{\varepsilon, \tau}^{(1)}$ does not change its sign. Henceforth, we can assume that $\psi_{\varepsilon, \tau}^{(1)} \rightarrow 1$ in $X$ as $\tau \rightarrow 0$.

Remark 3.5 If $\tau>0$ is small, then there is $\alpha>0$ such that $\left(\lambda-M_{\varepsilon, \tau} / \sigma\right)$ is invertible for $\lambda \in \Lambda_{\alpha}$. By Lemma 3.3, $\Lambda_{\alpha}$ is in the resolvent set of $M_{\varepsilon, \tau} / \sigma$ provided that $\alpha$ and $\tau$ are sufficiently small.

Lemma 3.6 For $N=1$, (A3) holds if (A3)' holds.

Proof Let $\hat{u}_{\varepsilon}=\xi_{\varepsilon}^{-\frac{q}{p-1}} u_{\varepsilon, 0}$, where $\hat{u}_{\varepsilon}$ and $\xi_{\varepsilon}$ satisfy (2.1). Multiplying the first equation of (2.1) by $\frac{d}{d x} \hat{u}_{\varepsilon, 0}$ and integrating it over $[0, x]$, we obtain

$$
\frac{\varepsilon^{2}}{2}\left(\frac{d \hat{u}_{\varepsilon}}{d x}\right)^{2}-\frac{\hat{u}_{\varepsilon}^{2}}{2}+\frac{\hat{u}_{\varepsilon}^{p+1}}{p+1}=-\frac{\left(\hat{u}_{\varepsilon}(0)\right)^{2}}{2}+\frac{\left(\hat{u}_{\varepsilon}(0)\right)^{p+1}}{p+1}
$$


Integrating (3.16) over $\Omega$, and dividing it by $\frac{1}{2} \int_{\Omega} \hat{u}_{\varepsilon}^{p+1} d x$, we have

$$
\frac{\int_{\Omega} \hat{u}_{\varepsilon}^{2} d x}{\int_{\Omega} \hat{u}_{\varepsilon}^{p+1} d x}=\frac{2}{p+1}+\frac{\varepsilon^{2} \int_{\Omega}\left(\frac{d \hat{u}_{\varepsilon}}{d x}\right)^{2} d x}{\int_{\Omega} \hat{u}_{\varepsilon}^{p+1} d x}+\frac{\left(1-2\left(\hat{u}_{\varepsilon}(0)\right)^{p-1} /(p+1)\right)\left(\hat{u}_{\varepsilon}(0)\right)^{2}}{\int_{\Omega} \hat{u}_{\varepsilon}^{p+1} d x} .
$$

By a phase plane analysis of $(2.1)$, it is easily to see that $0<\hat{u}_{\varepsilon}(0)<\left(\frac{p+1}{2}\right)^{\frac{1}{p-1}}$. Therefore, the third term of the right-hand side of (3.17) is positive. Furthermore, $\int_{\Omega} \hat{u}_{\varepsilon}^{2} d x / \int_{\Omega} \hat{u}_{\varepsilon}^{p+1} d x \geqslant 2 /(p+1)$, and hence (A3) holds provided that (A3)' holds.

Lemma 3.7 For $N=1$, (A2) holds if (A2)' holds.

Proof Let $\hat{u}_{\varepsilon}=\xi_{\varepsilon}^{-\frac{q}{p-1}} u_{\varepsilon, 0}$. Multiplying the first equation of (2.1) by $\hat{u}_{\varepsilon}^{p}$ and integrating it over $\Omega$, we have

$$
\varepsilon^{2} \int_{\Omega} \frac{d^{2} \hat{u}_{\varepsilon}}{d x^{2}} \hat{u}_{\varepsilon}^{p} d x-\int_{\Omega} \hat{u}_{\varepsilon}^{p+1} d x+\int_{\Omega} \hat{u}_{\varepsilon}^{2 p} d x=0
$$

Integrating by parts with the Neumann boundary condition for $\hat{u}_{\varepsilon}$, we have

$$
-p \varepsilon^{2} \int_{\Omega}\left(\frac{d \hat{u}_{\varepsilon}}{d x}\right)^{2} \hat{u}_{\varepsilon}^{p-1} d x-\int_{\Omega} \hat{u}_{\varepsilon}^{p+1} d x+\int_{\Omega} \hat{u}_{\varepsilon}^{2 p} d x=0 .
$$

Substituting (3.16) for the integrand of the first term of (3.19) gives

$$
\frac{3 p+1}{p+1} \int_{\Omega} \hat{u}_{\varepsilon}^{2 p} d x=(1+p) \int_{\Omega} \hat{u}_{\varepsilon}^{p+1} d x-p\left(1-\frac{2\left(\hat{u}_{\varepsilon}(0)\right)^{p-1}}{p+1}\right)\left(\hat{u}_{\varepsilon}(0)\right)^{2} \int_{\Omega} \hat{u}_{\varepsilon}^{p-1} d x .
$$

Therefore, we have that $\int_{\Omega} \hat{u}_{\varepsilon}^{2 p} d x / \int_{\Omega} \hat{u}_{\varepsilon}^{p+1} d x \leqslant(p+1)^{2} /(3 p+1)$, and hence (A2) holds provided that (A2)' holds.

\section{Spectral analysis of the operator $\mathscr{L}_{\varepsilon, 0}$}

In order to study the invertibility of $\mathscr{L}_{\varepsilon, 0}$, in this section we are mainly concerned with the invertibility of the operator $\left(A_{\varepsilon, 0, \lambda}-\lambda\right)$ defined by $(2.22)$ in $\S 2$.

Let $v_{\varepsilon, \tau}^{(n)}(n \geqslant 1)$ be the $n$th eigenvalue of $L_{\varepsilon, \tau}$ defined by (2.3) under the Neumann boundary condition repeated according to its multiplicity, and let $\phi_{\varepsilon, \tau}^{(n)}$ be the eigenfunction corresponding to $v_{\varepsilon, \tau}^{(n)}$. By $C$ we mean a constant which changes every line.

Lemma 4.1 Let $\lambda \in \mathbb{R}$. Suppose that (A3) holds. Then there exists $\alpha_{1}>0$ such that $\left(A_{\varepsilon, 0, \lambda}-\lambda\right)$ is invertible for $\lambda \in\left(-\alpha_{1}, v_{\varepsilon, 0}^{(1)}\right)$. In particular, $A_{\varepsilon, 0,0}$ is invertible.

Proof We assume that

$$
v_{\varepsilon, 0}^{(2)}<\lambda<v_{\varepsilon, 0}^{(1)}
$$

Note that $v_{\varepsilon, 0}^{(1)}>0$ and $v_{\varepsilon, 0}^{(2)}<0$ provided that $\varepsilon$ is small (see Corollary 2.7). We notice that the rank of $B_{\varepsilon, 0, \lambda}$ is one in the case where $\lambda$ is not an eigenvalue of $L_{\varepsilon, 0}$. Using the 
Sherman-Morrison formula, we can express $\left(A_{\varepsilon, 0, \lambda}-\lambda\right)^{-1}$ as (2.24). Here $h(\lambda) \in$ $C^{\infty}\left(\mathbb{R} \backslash\left\{v_{\varepsilon, 0}^{(1)}, v_{\varepsilon, 0}^{(2)}, \cdots\right\}\right)$ is

$$
h(\lambda)=1-\frac{q r}{(\sigma \lambda+1+s) \xi_{\varepsilon}^{q+s+1}}\left\langle\left(L_{\varepsilon, 0}-\lambda\right)^{-1}\left[u_{\varepsilon, 0}^{p}\right], u_{\varepsilon, 0}^{r-1}\right\rangle .
$$

By (4.1), $\lambda$ is not an eigenvalue of $L_{\varepsilon, 0}$, and hence $\left(L_{\varepsilon, 0}-\lambda\right)^{-1}$ exists. Therefore, when

$$
h(\lambda) \neq 0,
$$

the operator $\left(A_{\varepsilon, 0, \lambda}-\lambda\right)$ is invertible. Inequality (4.3) is equivalent to the inequality

$$
\sigma \lambda+1+s \neq \tilde{h}(\lambda)
$$

where $\tilde{h}(\lambda)=\frac{q r}{\xi_{\varepsilon}^{q+s+1}}\left\langle\left(L_{\varepsilon, 0}-\lambda\right)^{-1}\left[u_{\varepsilon, 0}^{p}\right], u_{\varepsilon, 0}^{r-1}\right\rangle$, and we will show that this holds for all $\lambda \in\left[0, v_{\varepsilon, 0}^{(1)}\right)$.

By Corollary 2.7 and Proposition 2.8, $L_{\varepsilon, 0}$ is invertible and we see by (3.1) that

$$
L_{\varepsilon, 0}^{-1}\left[u_{\varepsilon, 0}^{p}\right]=\frac{\xi_{\varepsilon}^{q}}{p-1} u_{\varepsilon, 0} .
$$

Using (4.5) and (3.2), we have that $\tilde{h}(0)=\frac{q r}{p-1}$. Therefore, by (1.2),

$$
\tilde{h}(0)>1+s \text {. }
$$

Next we will show that

$$
\frac{d}{d \lambda} \tilde{h}(\lambda) \geqslant \sigma \text { for } \lambda \in\left[0, v_{\varepsilon, 0}^{(1)}\right)
$$

Since $\left(L_{\varepsilon, 0}-\lambda\right)$ is self-adjoint, we see that $\left(\left(L_{\varepsilon, 0}-\lambda\right)^{-1}\right)^{*}=\left(\left(L_{\varepsilon, 0}-\lambda\right)^{*}\right)^{-1}=\left(L_{\varepsilon, 0}-\lambda\right)^{-1}$. Therefore, using (A0), we have

$$
\frac{d}{d \lambda} \tilde{h}(\lambda)=\frac{q r}{\xi_{\varepsilon}^{q+s+1}}\left\langle\left(L_{\varepsilon, 0}-\lambda\right)^{-2}\left[u_{\varepsilon, 0}^{p}\right], u_{\varepsilon, 0}^{r-1}\right\rangle=\frac{q r}{\xi_{\varepsilon}^{q+s+1}}\left\|\left(L_{\varepsilon, 0}-\lambda\right)^{-1}\left[u_{\varepsilon, 0}^{p}\right]\right\|^{2} .
$$

From the eigenfunction expansion of $\left(L_{\varepsilon, 0}-\lambda\right)^{-1}$, we have

$$
\left\|\left(L_{\varepsilon, 0}-\lambda\right)^{-1}\left[u_{\varepsilon, 0}^{p}\right]\right\|_{X}^{2}=\frac{\xi_{\varepsilon}^{2 q}}{(p-1)^{2}} \sum_{n \geqslant 1}\left(\frac{v_{\varepsilon, 0}^{(n)}}{v_{\varepsilon, 0}^{(n)}-\lambda}\right)^{2}\left\langle u_{\varepsilon, 0}, \phi_{\varepsilon, 0}^{(n)}\right\rangle^{2} .
$$

Now we see that

$$
\inf \left\{\left(\frac{v_{\varepsilon, 0}^{(n)}}{v_{\varepsilon, 0}^{(n)}-\lambda}\right)^{2} \mid n \geqslant 1, v_{\varepsilon, 0}^{(2)}<\lambda<v_{\varepsilon, 0}^{(1)}\right\}=\kappa,
$$

where $\kappa$ is defined in Theorem B. Thus we obtain

$$
\frac{d}{d \lambda} \tilde{h}(\lambda) \geqslant \frac{q r \kappa}{(p-1)^{2} \xi_{\varepsilon}^{-q+s+1}} \sum_{n \geqslant 1}\left\langle u_{\varepsilon, 0}, \phi_{\varepsilon, 0}^{(n)}\right\rangle^{2}=\frac{q r \kappa\left\|u_{\varepsilon, 0}\right\|_{X}^{2}}{(p-1)^{2} \xi_{\varepsilon}^{-q+s+1}} .
$$

If condition (A3) holds, then (4.7) holds as well. Owing to (4.6) and (4.7), the inequality (4.4) holds for all $\lambda \in\left[0, v_{\varepsilon, 0}^{(1)}\right)$. The proof is complete. 
Lemma 4.2 Let $\lambda \in \mathbb{R}$. The operator $\left(A_{\varepsilon, 0, \lambda}-\lambda\right)$ is invertible for all $\lambda \in\left(v_{\varepsilon, 0}^{(1)},+\infty\right)$.

Proof We use the notation as in the proof of Lemma 4.1. We show that (4.4) holds for $\lambda \in\left(v_{\varepsilon, 0}^{(1)},+\infty\right)$. Using the eigenfunction expansion to $\left(L_{\varepsilon, 0}-\lambda\right)^{-1}[\cdot]$, we have

$$
\left\langle\left(L_{\varepsilon, 0}-\lambda\right)^{-1}\left[u_{\varepsilon, 0}^{p}\right], u_{\varepsilon, 0}^{r-1}\right\rangle=\sum_{n \geqslant 1} \frac{\left\langle u_{\varepsilon, 0}^{p}, \phi_{\varepsilon, 0}^{(n)}\right\rangle^{2}}{v_{\varepsilon, 0}^{(n)}-\lambda} .
$$

Since $\lambda>v_{\varepsilon, 0}^{(1)}$, the right-hand side of (4.8) is negative, and the right-hand side of (4.4) is negative as well. However, the left-hand side of (4.4) is positive for $\lambda \in\left(v_{\varepsilon, 0}^{(1)},+\infty\right)$. Therefore (4.4) holds for $\lambda \in\left(v_{\varepsilon, 0}^{(1)},+\infty\right)$.

Lemma 4.3 The operator $\left(A_{\varepsilon, 0, v_{\varepsilon, 0}^{(1)}}-v_{\varepsilon, 0}^{(1)}\right)$ is invertible.

Proof Let $f \in X$, and $w=\rho \phi_{\varepsilon, 0}^{(1)}+w^{\perp}$, where

$$
\rho=\frac{\left\langle f, \phi_{\varepsilon, 0}^{(1)}\right\rangle-\left\langle B_{\varepsilon, 0, v_{\varepsilon, 0}^{(1)}}\left[w^{\perp}\right], \phi_{\varepsilon, 0}^{(1)}\right\rangle}{\left\langle B_{\varepsilon, 0, v_{\varepsilon, 0}^{(1)}}\left[\phi_{\varepsilon, 0}^{(1)}\right], \phi_{\varepsilon, 0}^{(1)}\right\rangle}, w^{\perp}=\left(L_{\varepsilon, 0}-v_{\varepsilon, 0}^{(1)}\right)^{-1}\left[f-\frac{\left\langle f, \phi_{\varepsilon, 0}^{(1)}\right\rangle}{\left\langle u_{\varepsilon, 0}^{p}, \phi_{\varepsilon, 0}^{(1)}\right\rangle} u_{\varepsilon, 0}^{p}\right] .
$$

Note that $\phi_{\varepsilon, 0}^{(1)}$ is positive, and that $<B_{\varepsilon, 0, v_{\varepsilon, 0}^{(1)}}\left[\phi_{\varepsilon, 0}^{(1)}\right], \phi_{\varepsilon, 0}^{(1)}>=\frac{q r<u_{\varepsilon, 0}^{p}, \phi_{\varepsilon, 0}^{(1)}>^{2}}{\left(\sigma v_{\varepsilon, 0}^{(1)}+1+s\right)_{\varepsilon}^{q+s+1}} \neq 0$. The function $w$ is a solution for $\left(A_{\varepsilon, 0, v_{\varepsilon, 0}^{(1)}}-v_{\varepsilon, 0}^{(1)}\right) w=f$, and there is a constant $C>0$ such that $\|w\|_{Y}<C\|f\|_{X}$.

Lemma 4.4 Let $\lambda \in \mathbb{C} \backslash \mathbb{R}$. Suppose that (A1) holds. Then there exists a constant $\alpha_{2}>0$ such that the operator $\left(A_{\varepsilon, 0, \lambda}-\lambda\right)$ is invertible for $\lambda \in \Lambda_{\alpha_{2}} \backslash \mathbb{R}$.

Proof Since the spectrum of $\left(A_{\varepsilon, 0, \lambda}-\lambda\right)$ consists only of the eigenvalues, we have to show that there is no eigenvalue in $\Lambda_{\alpha_{2}} \backslash \mathbb{R}$.

Let $w=w_{R}+i w_{I}$ and $\lambda=a+b i$. We assume that $w$ and $\lambda$ satisfy

$$
\left(A_{\varepsilon, 0, \lambda}-\lambda\right) w=0 .
$$

This is equivalent to

$$
L_{\varepsilon, 0}\left[w_{R}+i w_{I}\right]-\frac{q r}{\sigma(a+b i)+1+s}\left\langle w_{R}+i w_{I}, u_{\varepsilon, 0}^{p}\right\rangle \frac{u_{\varepsilon, 0}^{p}}{\xi_{\varepsilon}^{q+s+1}}=(a+b i)\left(w_{R}+i w_{I}\right) .
$$

That is, we have an equation

$$
\begin{aligned}
L_{\varepsilon, 0} w_{R}- & \frac{q r(a \sigma+1+s) u_{\varepsilon, 0}^{p}}{\left((a \sigma+1+s)^{2}+(b \sigma)^{2}\right) \xi_{\varepsilon}^{q+s+1}}\left\langle w_{R}, u_{\varepsilon, 0}^{p}\right\rangle \\
& -\frac{q r b \sigma u_{\varepsilon, 0}^{p}}{\left((a \sigma+1+s)^{2}+(b \sigma)^{2}\right) \xi_{\varepsilon}^{q+s+1}}\left\langle w_{I}, u_{\varepsilon, 0}^{p}\right\rangle=a w_{R}-b w_{I},
\end{aligned}
$$


for the real part, and an equation

$$
\begin{aligned}
L_{\varepsilon, 0} w_{I}- & \frac{q r(a \sigma+1+s) u_{\varepsilon, 0}^{p}}{\left((a \sigma+1+s)^{2}+(b \sigma)^{2}\right) \xi_{\varepsilon}^{q+s+1}}\left\langle w_{I}, u_{\varepsilon, 0}^{p}\right\rangle \\
& +\frac{q r b \sigma u_{\varepsilon, 0}^{p}}{\left((a \sigma+1+s)^{2}+(b \sigma)^{2}\right) \xi_{\varepsilon}^{q+s+1}}\left\langle w_{R}, u_{\varepsilon, 0}^{p}\right\rangle=b w_{R}+a w_{I},
\end{aligned}
$$

for the imaginary part. The equation $\left\langle(4.11), w_{R}\right\rangle+\left\langle(4.12), w_{I}\right\rangle$ gives

$$
\begin{aligned}
\left\langle L_{\varepsilon, 0} w_{R}, w_{R}\right\rangle+ & \left\langle L_{\varepsilon, 0} w_{I}, w_{I}\right\rangle-a\left(\left\langle w_{R}, w_{R}\right\rangle+\left\langle w_{I}, w_{I}\right\rangle\right) \\
& =\frac{q r(a \sigma+1+s)}{\left((a \sigma+1+s)^{2}+(b \sigma)^{2}\right) \xi_{\varepsilon}^{q+s+1}}\left(\left\langle w_{R}, u_{\varepsilon, 0}^{p}\right\rangle^{2}+\left\langle w_{I}, u_{\varepsilon, 0}^{p}\right\rangle^{2}\right) .
\end{aligned}
$$

The equation $\left\langle(4.12), w_{R}\right\rangle-\left\langle(4.11), w_{I}\right\rangle$ gives

$$
\begin{aligned}
\frac{q r b \sigma}{\left((a \sigma+1+s)^{2}+(b \sigma)^{2}\right) \xi_{\varepsilon}^{q+s+1}} & \left(\left\langle w_{R}, u_{\varepsilon, 0}^{p}\right\rangle^{2}+\left\langle w_{I}, u_{\varepsilon, 0}^{p}\right\rangle^{2}\right) \\
& =b\left(\left\langle w_{R}, w_{R}\right\rangle+\left\langle w_{I}, w_{I}\right\rangle\right) .
\end{aligned}
$$

By the assumption of this lemma, $b \neq 0$. Multiplying (4.14) by $(a \sigma+1+s) /(b \sigma)$ leads to

$$
\begin{aligned}
\frac{q r(a \sigma+1+s)}{\left((a \sigma+1+s)^{2}+(b \sigma)^{2}\right) \xi_{\varepsilon}^{q+s+1}} & \left(\left\langle w_{R}, u_{\varepsilon, 0}^{p}\right\rangle^{2}+\left\langle w_{I}, u_{\varepsilon, 0}^{p}\right\rangle^{2}\right) \\
& =\left(a+\frac{1+s}{\sigma}\right)\left(\left\langle w_{R}, w_{R}\right\rangle+\left\langle w_{I}, w_{I}\right\rangle\right) .
\end{aligned}
$$

Adding (4.13) and (4.15) yeilds

$$
\left\langle L_{\varepsilon, 0} w_{R}, w_{R}\right\rangle+\left\langle L_{\varepsilon, 0} w_{I}, w_{I}\right\rangle=\left(2 a+\frac{1+s}{\sigma}\right)\left(\left\langle w_{R}, w_{R}\right\rangle+\left\langle w_{I}, w_{I}\right\rangle\right) .
$$

If $\left\|w_{R}+i w_{I}\right\|^{2}=\left\|w_{R}\right\|^{2}+\left\|w_{I}\right\|^{2}=1$, then

$$
2 a=-\frac{1+s}{\sigma}+\left\langle L_{\varepsilon, 0} w_{R}, w_{R}\right\rangle+\left\langle L_{\varepsilon, 0} w_{I}, w_{I}\right\rangle \leqslant-\frac{1+s}{\sigma}+v_{\varepsilon, 0}^{(1)} .
$$

Because of (A1), we see that $a<0$. The proof is complete.

Lemma 4.5 Let $\lambda \in \mathbb{R}$. Suppose that (A1) and (A3) hold. Then there exist $\alpha_{3}>0$ and $\alpha_{4}>0$ such that

$$
\left\langle\left(\lambda-A_{\varepsilon, 0, \lambda}\right) w, w\right\rangle \geqslant \alpha_{3}\|w\|_{X}^{2} \quad \text { for } \quad \lambda \in\left(-\alpha_{4},+\infty\right) \text { and } w \in Y
$$

Proof By Lemmas 4.1, 4.2 and 4.3, there exists a constant $\alpha_{4}>0$ such that for $\lambda \in\left[-\alpha_{4},+\infty\right)$ zero is not an eigenvalue of $\left(\lambda-A_{\varepsilon, 0, \lambda}\right)$. Since $\left\|B_{\varepsilon, 0, \lambda}\right\|_{\mathscr{L}(X, X)}$ is bounded for $\lambda \in\left[-\alpha_{4},+\infty\right)$, and $\left(\lambda-A_{\varepsilon, 0, \lambda}\right)=\left(\lambda-L_{\varepsilon, 0}-B_{\varepsilon, 0, \lambda}\right)$, the first eigenvalue of the operator $\left(\lambda-A_{\varepsilon, 0, \lambda}\right)$ tends to $+\infty$ as $\lambda \rightarrow+\infty$. The continuity of the first eigenvalue with 
respect to $\lambda$, together with the above two facts, says that for $\lambda \in\left(-\alpha_{4},+\infty\right)$ the first eigenvalue is positive and uniformly away from zero. Therefore, there is a constant $\alpha_{3}>0$ such that $\left(\lambda-\alpha_{3}-A_{\varepsilon, 0, \lambda}\right)$ is a positive operator for $\lambda \in\left(-\alpha_{4},+\infty\right)$. The proof is complete.

Lemma 4.6 Let $\lambda \in \mathbb{C}$, and $w=w_{R}+i w_{I}$. Suppose that (A1), (A2) and (A3) hold. Then there exist $\alpha_{5}>0$ and $\alpha_{6}>0$ such that

$$
\left\|\left(A_{\varepsilon, 0, \lambda}-\lambda\right)^{-1} w\right\|_{X} \leqslant \alpha_{5}\|w\|_{X} \quad \text { for } \lambda \in \Lambda_{\alpha_{6}} .
$$

Proof Let $\lambda=a+b i$ and let $\delta_{1}>0$ be small. We consider two cases seperately.

Case 1; for $(a, b) \in \Lambda^{(1)}:=\left\{(a, b) \mid b^{2} \sigma \leqslant \delta_{1}(a \sigma+1+s)\right\}$, where $\delta_{1}$ will be defined later. Substituting $\lambda=a+b i$ and $w=w_{R}+i w_{I}$ into $\left\langle\left(\lambda-A_{\varepsilon, 0, \lambda}\right) w, w\right\rangle$, we have

$$
\left\langle\left(a+b i-A_{\varepsilon, 0, a+b i}\right)\left[w_{R}+i w_{I}\right], w_{R}+i w_{I}\right\rangle=\chi+i \omega .
$$

Here

$$
\begin{aligned}
\chi:= & -\left\langle L_{\varepsilon, 0} w_{R}, w_{R}\right\rangle-\left\langle L_{\varepsilon, 0} w_{I}, w_{I}\right\rangle+a\left(\left\langle w_{R}, w_{R}\right\rangle+\left\langle w_{I}, w_{I}\right\rangle\right) \\
& +\frac{q r(a \sigma+1+s)}{\left((a \sigma+1+s)^{2}+(b \sigma)^{2}\right) \xi_{\varepsilon}^{q+s+1}}\left(\left\langle w_{R}, u_{\varepsilon, 0}^{p}\right\rangle^{2}+\left\langle w_{I}, u_{\varepsilon, 0}^{p}\right\rangle^{2}\right),
\end{aligned}
$$

and

$$
\begin{aligned}
\omega:= & \frac{q r b \sigma}{\left((a \sigma+1+s)^{2}+(b \sigma)^{2}\right) \xi_{\varepsilon}^{q+s+1}}\left(\left\langle w_{R}, u_{\varepsilon, 0}^{p}\right\rangle^{2}+\left\langle w_{I}, u_{\varepsilon, 0}^{p}\right\rangle^{2}\right) \\
& -b\left(\left\langle w_{R}, w_{R}\right\rangle+\left\langle w_{I}, w_{I}\right\rangle\right) .
\end{aligned}
$$

By Lemma 4.5 , there exist $\delta_{2}>0$ and $\delta_{3}>0$ such that

$$
\left\langle\left(a+\beta-A_{\varepsilon, 0, a+\beta}\right) w_{R}, w_{R}\right\rangle \geqslant \delta_{2}\left\|w_{R}\right\|_{X}^{2} \quad \text { for } a \in\left(-\delta_{3},+\infty\right),
$$

where $\beta=\left(b^{2} \sigma\right) /(a \sigma+1+s)$. The constant $\delta_{1}$ can be now taken to ensure $\delta_{2}>\delta_{1}$. Thus we see that

$$
\begin{aligned}
\chi & =\left\langle\left(a-A_{\varepsilon, 0, a+\beta}\right) w_{R}, w_{R}\right\rangle+\left\langle\left(a-A_{\varepsilon, 0, a+\beta} w_{I}, w_{I}\right\rangle\right. \\
& \geqslant\left(\delta_{2}-\delta_{1}\right)\left(\left\|w_{R}\right\|_{X}^{2}+\left\|w_{I}\right\|_{X}^{2}\right) .
\end{aligned}
$$

Therefore,

$$
\begin{aligned}
\left|\left\langle\left(a+b i-A_{\varepsilon, 0, a+b i}\right)\left[w_{R}+i w_{I}\right], w_{R}+i w_{I}\right\rangle\right| & \geqslant \sqrt{\chi^{2}+\omega^{2}} \geqslant \chi \\
& \geqslant\left(\delta_{2}-\delta_{1}\right)\left\|w_{R}+i w_{I}\right\|_{X}^{2}
\end{aligned}
$$

for $(a, b) \in \Lambda^{(1)}$, which indicates that

$$
\left\|\left(a+b i-A_{\varepsilon, 0, a+b i}\right)\left[w_{R}+i w_{I}\right]\right\|_{X} \geqslant C\left\|w_{R}+i w_{I}\right\|_{X} \quad \text { for }(a, b) \in \Lambda^{(1)} .
$$


Case 2; for $(a, b) \in \Lambda^{(2)}:=\left\{(a, b)|2 \sqrt{\sigma}| b \mid>\sqrt{\delta_{1}}, a>-\delta_{4}\right\}$, where $\delta_{4}$ is to be defined later.

Firstly, we note that

$$
\begin{gathered}
\frac{q r \sigma}{\left((a \sigma+1+s)^{2}+(b \sigma)^{2}\right) \xi_{\varepsilon}^{q+s+1}}\left(\left\langle w_{R}, u_{\varepsilon, 0}^{p}\right\rangle^{2}+\left\langle w_{I}, u_{\varepsilon, 0}^{p}\right\rangle^{2}\right) \\
\leqslant \frac{q r \sigma\left\|u_{\varepsilon, 0}^{p}\right\|_{X}^{2}}{\left((a \sigma+1+s)^{2}+(b \sigma)^{2}\right) \xi_{\varepsilon}^{q+s+1}}\left(\left\|w_{R}\right\|_{X}^{2}+\left\|w_{I}\right\|_{X}^{2}\right) .
\end{gathered}
$$

By (3.3) and (A2), we see that there exists a constant $\delta_{4}>0$ such that

$$
\frac{q r \sigma\left\|u_{\varepsilon, 0}^{p}\right\|_{X}^{2}}{\left((a \sigma+1+s)^{2}+(b \sigma)^{2}\right) \xi_{\varepsilon}^{q+s+1}}<1 \quad \text { for }(a, b) \in \Lambda^{(2)} .
$$

Therefore, there exists a constant $\delta_{5} \in(0,1)$ such that

$$
\frac{q r \sigma\left\|u_{\varepsilon, 0}^{p}\right\|_{X}^{2}}{\left((a \sigma+1+s)^{2}+(b \sigma)^{2}\right) \xi_{\varepsilon}^{q+s+1}}<\delta_{5} \quad \text { for }(a, b) \in \Lambda^{(2)} .
$$

Hence, we see that

$$
\begin{aligned}
|\omega|= & b\left(\left\|w_{R}\right\|_{X}^{2}-\frac{q r \sigma\left\langle w_{R}, u_{\varepsilon, \tau}^{p}\right\rangle}{\left((a \sigma+1+s)^{2}+(b \sigma)^{2}\right) \xi_{\varepsilon}^{q+s+1}}\right) \\
& +b\left(\left\|w_{I}\right\|_{X}^{2}-\frac{q r \sigma\left\langle w_{I}, u_{\varepsilon, \tau}^{p}\right\rangle}{\left((a \sigma+1+s)^{2}+(b \sigma)^{2}\right) \xi_{\varepsilon}^{q+s+1}}\right) \\
\geqslant & C\left(\left\|w_{R}\right\|_{X}^{2}+\left\|w_{I}\right\|_{X}^{2}\right) .
\end{aligned}
$$

Thus we obtain

$$
\left\|\left(a+b i-A_{\varepsilon, 0, a+b i}\right)\left[w_{R}+i w_{I}\right]\right\|_{X} \geqslant C\left\|w_{R}+i w_{I}\right\|_{X} \quad \text { for }(a, b) \in \Lambda^{(2)} .
$$

Since there exists $\alpha_{6}>0$ such that $\Lambda_{\alpha_{6}} \subset \Lambda^{(1)} \cup \Lambda^{(2)}$, (4.17) follows from (4.18) and (4.19).

Proof of Theorem A Let $\left(u_{\varepsilon, 0}, v_{\varepsilon, 0}\right)$ be a solution of the shadow system (1.5) given by Proposition 2.1. We show that $D_{(u, \xi, \zeta)} \mathscr{F}\left(\varepsilon, 0, u_{\varepsilon, 0}, v_{\varepsilon, 0}, 0\right)$ is invertible, as stated in $\S 2.1$. If we prove that, then the proof is complete.

Linearizing $\mathscr{F}$ at $\left(u_{\varepsilon, 0}, v_{\varepsilon, 0}\right)$, we obtain

$$
D_{(u, \xi, \zeta)} \mathscr{F}=\left(\begin{array}{ccc} 
& & -\frac{q u_{\varepsilon}^{p}}{\xi_{\varepsilon}^{q+1}} \\
\mathscr{L}_{\varepsilon, 0} & -1-s \\
0 & 0 & \Delta
\end{array}\right),
$$


where $\mathscr{L}_{\varepsilon, 0}$ is defined in $\S 2.2$. Here $\Delta: C_{N}^{2+d} \rightarrow Z$ is a homeomorphism, and $Z$ is defined in Subsection 2.1. If $\mathscr{L}_{\varepsilon, 0}$ is invertible, then $D_{(u, \xi, \zeta)} \mathscr{F}$ is invertible as well. By Lemma 4.1, $A_{\varepsilon, 0,0}$ is invertible, and therefore $\mathscr{L}_{\varepsilon, 0}$ is invertible as well (for the details, see $\S 2.2$ ).

Proof of Theorem $\mathbf{C}$ Let $\alpha^{*}=\min \left\{\alpha_{1}, \alpha_{2}, \alpha_{4}, \alpha_{6}\right\}$. If (A1), (A2) and (A3) hold, then all Lemmas of $\S 4$ hold for $\lambda \in \Lambda_{\alpha^{*}}$. Then $\left(A_{\varepsilon, 0, \lambda}-\lambda\right)$ is invertible for all $\lambda \in \Lambda_{\alpha^{*}}$, which implies that $\left(\mathscr{L}_{\varepsilon, 0}-\lambda\right)$ is also invertible for $\lambda \in \Lambda_{\alpha^{*}}$. Therefore, the boundary spike layer $\left(u_{\varepsilon, 0}, v_{\varepsilon, 0}\right)$ is stable. In particular, if $\sigma$ is small, then (A1), (A2) and (A3) are automatically satisfied. The proof is complete.

\section{Spectral analysis of the operator $\mathscr{L}_{\varepsilon, \tau}$}

We will now show that $A_{\varepsilon, \tau, \lambda}$ is close to $A_{\varepsilon, 0, \lambda}$ in the sense of the operator norm provided that $\tau$ is sufficiently small. Let $D_{\varepsilon, \tau, \lambda}=A_{\varepsilon, \tau, \lambda}-A_{\varepsilon, 0, \lambda}$. Then the following Lemma holds.

Lemma 5.1 Let $\lambda \in \Lambda_{\alpha^{*}}$. Then

$$
\lim _{\tau \rightarrow 0}\left\|D_{\varepsilon, \tau, \lambda}\right\|_{\mathscr{L}(X, X)}=0
$$

The convergence is uniform for $\lambda \in \Lambda_{\alpha^{*}}$.

Proof We divide $D_{\varepsilon, \tau, \lambda}$ into three parts,

$$
\begin{aligned}
D_{\varepsilon, \tau, \lambda} & =\left(B_{\varepsilon, \tau, \lambda}-B_{\varepsilon, 0, \lambda}\right)+R_{\varepsilon, \tau, \lambda}+\left(L_{\varepsilon, \tau}-L_{\varepsilon, 0}\right) \\
& =D_{\varepsilon, \tau, \lambda}^{(1)}+D_{\varepsilon, \tau, \lambda}^{(2)}+D_{\varepsilon, \tau}^{(3)} .
\end{aligned}
$$

Firstly, we will show that

$$
\lim _{\tau \rightarrow 0}\left\|D_{\varepsilon, \tau, \lambda}^{(2)}\right\|_{\mathscr{L}(X, X)}=0 \quad \text { uniformly for } \lambda \in \Lambda_{\alpha^{*}} .
$$

Here $R_{\varepsilon, \tau, \lambda}\left(=D_{\varepsilon, \tau, \lambda}^{(2)}\right)$ is defined by (2.16). Then

$$
\begin{aligned}
\left\|D_{\varepsilon, \tau, \lambda}^{(2)} w\right\|_{X} & \leqslant\left\|q r \frac{u_{\varepsilon, \tau}^{p}}{v_{\varepsilon, \tau}^{q+1}}\left(\sigma \lambda-M_{\varepsilon, \tau}\right)^{\dagger}\left[\frac{u_{\varepsilon, \tau}^{r-1}}{v_{\varepsilon, \tau}^{s}} w\right]\right\|_{X} \\
& \leqslant\left\|q r \frac{u_{\varepsilon, \tau}^{p}}{v_{\varepsilon, \tau}^{q+1}}\right\|_{L^{\infty}}\left\|\left(\sigma \lambda-M_{\varepsilon, \tau}\right)^{\dagger}\right\|_{\mathscr{L}(X, X)}\left\|\frac{u_{\varepsilon, \tau}^{r-1}}{v_{\varepsilon, \tau}^{s}}\right\|_{L^{\infty}}\|w\|_{X} .
\end{aligned}
$$

We can see (Remark 2.3) that there is a constant $C>0$ such that $\left\|u_{\varepsilon, \tau}^{p} / v_{\varepsilon, \tau}^{q+1}\right\|_{L^{\infty}}<C$ and $\left\|u_{\varepsilon, \tau}^{r-1} / v_{\varepsilon, \tau}^{s}\right\|_{L^{\infty}}<C$ for small $\tau$. Then by (2.14), we see that (5.3) holds.

Next, we show that

$$
\lim _{\tau \rightarrow 0}\left\|D_{\varepsilon, \tau, \lambda}^{(1)}\right\|_{\mathscr{L}(X, X)}=0 \quad \text { uniformly for } \lambda \in \Lambda_{\alpha^{*}}
$$


Here $B_{\varepsilon, \tau, \lambda}-B_{\varepsilon, 0, \lambda}\left(=D_{\varepsilon, \tau, \lambda}^{(1)}\right)$ defined by (2.30). We have

$$
\begin{aligned}
& D_{\varepsilon, \tau, \lambda}^{(1)} w=\frac{q u_{\varepsilon, \tau}^{p}}{\left(\sigma \lambda-\sigma \mu_{\varepsilon, \tau}^{(1)}\right) v_{\varepsilon, \tau}^{q+1}}\left\langle\frac{r u_{\varepsilon, \tau}^{r-1} w}{v_{\varepsilon, \tau}^{s}}, \psi_{\varepsilon, \tau}^{(1)}\right\rangle\left(\psi_{\varepsilon, \tau}^{(1)}-1\right) \\
& +\frac{q u_{\varepsilon, \tau}^{p}}{\left(\sigma \lambda-\sigma \mu_{\varepsilon, \tau}^{(1)}\right) v_{\varepsilon, \tau}^{q+1}}\left\langle\frac{r u_{\varepsilon, \tau}^{r-1} w}{v_{\varepsilon, \tau}^{s}}, \psi_{\varepsilon, \tau}^{(1)}-1\right\rangle+\frac{q u_{\varepsilon, \tau}^{p}}{\left(\sigma \lambda-\sigma \mu_{\varepsilon, \tau}^{(1)}\right) v_{\varepsilon, \tau}^{q+1}}\left\langle r u_{\varepsilon, \tau}^{r-1} w\left(\frac{1}{v_{\varepsilon, \tau}^{s}}-\frac{1}{\xi_{\varepsilon}^{s}}\right), 1\right\rangle \\
& +\frac{q u_{\varepsilon, \tau}^{p}}{\left(\sigma \lambda-\sigma \mu_{\varepsilon, \tau}^{(1)}\right) v_{\varepsilon, \tau}^{q+1}}\left\langle\frac{r w}{\xi_{\varepsilon}^{s}}\left(u_{\varepsilon, \tau}^{r-1}-u_{\varepsilon, 0}^{r-1}\right), 1\right\rangle+\frac{q\left(u_{\varepsilon, \tau}^{p}-u_{\varepsilon, 0}^{p}\right)}{\left(\sigma \lambda-\sigma \mu_{\varepsilon, \tau}^{(1)}\right) v_{\varepsilon, \tau}^{q+1}}\left\langle\frac{r u_{\varepsilon, 0}^{r-1} w}{\xi_{\varepsilon}^{s}}, 1\right\rangle \\
& +\frac{q u_{\varepsilon, 0}^{p}}{\sigma \lambda-\sigma \mu_{\varepsilon, \tau}^{(1)}}\left(\frac{1}{v_{\varepsilon, \tau}^{q+1}}-\frac{1}{\xi_{\varepsilon}^{q+1}}\right)\left\langle\frac{r u_{\varepsilon, 0}^{r-1} w}{\xi_{\varepsilon}^{s}}, 1\right\rangle \\
& +\left(\frac{1}{\sigma \lambda-\sigma \mu_{\varepsilon, \tau}^{(1)}}-\frac{1}{\sigma \lambda+1+s}\right) \frac{q u_{\varepsilon, 0}^{p}}{\xi_{\varepsilon}^{q+1}}\left\langle\frac{r u_{\varepsilon, 0}^{r-1} w}{\xi_{\varepsilon}^{s}}, 1\right\rangle .
\end{aligned}
$$

By Lemma 3.3 (i), the first and second terms tend to zero as $\tau \rightarrow 0$. Using Remark 2.3, we see that the third, forth, fifth and sixth terms tend to zero as $\tau \rightarrow 0$. By Lemma 3.3 (ii), the seventh term tends to zero in $X$. Therefore, (5.4) holds.

We show that

$$
\lim _{\tau \rightarrow 0}\left\|D_{\varepsilon, \tau}^{(3)}\right\|_{\mathscr{L}(X, X)}=0 .
$$

Here $L_{\varepsilon, \tau}-L_{\varepsilon, 0}\left(=D_{\varepsilon, \tau}^{(3)}\right)$ is defined as (2.29). It is clear from (2.29) and Remark 2.3 that (5.5) holds.

Because of (5.3), (5.4) and (5.5), the limit (5.1) holds.

Lemma 5.2 Let $\lambda \in \mathbb{C}$. If all the assumptions of Lemma 4.6 are satisfied, then there exists $\tau_{0}>0$ such that if $\tau \in\left(0, \tau_{0}\right)$, then $\left(A_{\varepsilon, \tau, \lambda}-\lambda\right)$ is invertible for all $\lambda \in \Lambda_{\alpha^{*}}$.

Proof Since $A_{\varepsilon, \tau, \lambda}=L_{\varepsilon, \tau}+B_{\varepsilon, \tau, \lambda}$ and $A_{\varepsilon, 0, \lambda}=L_{\varepsilon, 0}+B_{\varepsilon, 0, \lambda}$, we have

$$
A_{\varepsilon, \tau, \lambda}-\lambda=A_{\varepsilon, 0, \lambda}-\lambda+D_{\varepsilon, \tau, \lambda}
$$

where $D_{\varepsilon, \tau, \lambda}=A_{\varepsilon, \tau, \lambda}-A_{\varepsilon, 0, \lambda}$. Let $T=\left(A_{\varepsilon, 0, \lambda}-\lambda\right)^{-1} D_{\varepsilon, \tau, \lambda}$. Then (2.31) hold. Since $\left(A_{\varepsilon, 0, \lambda}-\lambda\right)$ is invertible for $\lambda \in \Lambda_{\alpha^{*}}$, we show that $(I+T)$ is invertible as well. Now, we have the inequality

$$
\|T\|_{\mathscr{L}(X, X)} \leqslant\left\|\left(A_{\varepsilon, 0, \lambda}-\lambda\right)^{-1}\right\|_{\mathscr{L}(X, X)} \cdot\left\|D_{\varepsilon, \tau, \lambda}\right\|_{\mathscr{L}(X, X)} \cdot
$$

By (4.17) and (5.1), the right-hand side of this converges to zero as $\tau \rightarrow 0$, and this convergence is uniform for $\lambda \in \Lambda_{\alpha^{*}}$. Therefore, there exists $\tau_{0}>0$ such that

$$
\|T\|_{\mathscr{L}(X, X)}<\frac{1}{2} \text { for } \tau \in\left(0, \tau_{0}\right) \text { and } \lambda \in \Lambda_{\alpha^{*}}
$$


In this case $(I+T)$ is invertible and the inverse can be expressed by the Neumann series

$$
(I+T)^{-1}=\sum_{n=0}^{\infty}(-T)^{n}=I-T+T^{2}-T^{3}+T^{4}-T^{5}+\cdots,
$$

where $(-T)^{0}:=I$. The proof is complete.

By (2.8), (2.9) and (2.10), the following Lemma holds.

Lemma 5.3 Let $\lambda \in \mathbb{C}$. If $\left(A_{\varepsilon, \tau, \lambda}-\lambda\right)$ and $\left(\lambda-M_{\varepsilon, \tau} / \sigma\right)$ are invertible, then the operator $\left(\mathscr{L}_{\varepsilon, \tau}-\lambda\right)$ is invertible.

Proof of Theorem $\mathbf{B}$ If (A1), (A2) and (A3) hold, then $\left(A_{\varepsilon, 0, \lambda}-\lambda\right)$ is invertible for $\lambda \in \Lambda_{\alpha^{\circ}}$. For small $\tau_{0}$, if $\tau<\tau_{0}$, then $\left(A_{\varepsilon, \tau, \lambda}-\lambda\right)$ is invertible for $\lambda \in \Lambda_{\alpha^{*}}$. Lemma 5.3 claims that $\left(\mathscr{L}_{\varepsilon, \tau}-\lambda\right)$ is invertible for $\lambda \in \Lambda_{\alpha^{*}}$. In particular, (A1), (A2) and (A3) are automatically satisfied provided that $\sigma$ is small. We see by Lemmas 3.6 and 3.7 that (A2) and (A3) hold if (A2)' and (A3)' hold respectively. Thus the proof is complete.

\section{Conclusions}

By perturbing a boundary (one-)spike layer for the shadow system, we showed that a bounady (one-)spike layer for the full Gierer-Meinhardt system exists.

Some natural questions arise: firstly, we assumed that $P_{0} \in \partial \Omega$, which is the maximum point of the boundary (one-)spike layer, is a non-degenerate local maximum point of $\partial \Omega$. The assumption seems to be technical. It would be desirable to remove this assumption. Secondly, the assumption (A0) appears to be restrictive. Because of this assumption, the case of the original Gierer-Meinhardt system is not included. The method of the proof used in this paper depends highly on the self-adjointness of the linear operator $A_{\varepsilon, 0, \lambda}$ $(\lambda \in \mathbb{R})$. Therefore, we may need an idea for more general case where the assumption (A0) does not hold. Thirdly, is there any stable boundary multispike layer for the full system? It appears that we have to study the eigenvalues of the linear operator $\varepsilon^{2} \Delta-1+p u^{p-1}$, where $u$ is a boundary multispike layer for $\varepsilon^{2} \Delta u-u+u^{p}=0$.

It would be also interesting to clarify the difference and similarity between the shadow system and the full system.

\section{Acknowledgements}

The author thanks Professors Y. Nishiura and A. Korobeinikov for thier helpful comments, and the referees for their careful reviewing of the manuscript.

\section{References}

[1] De Groen, P. \& Karadzhov, G. (2002) Metastability of the steady state spike solutions in the shadow system for Gierer-Meinhardt's equations. C. R. Acad. Bulgare Sci. 55 (1), 13-18. 
[2] Del Pino, M., Felmer, P. L. \& KowalczyK, M. (2002) Boundary spikes in the GiererMeinhardt system. Commun. Pure Appl. Anal. 1 (4), 437-456.

[3] Del Pino, M., Felmer, P. L. \& Wei, J. (1999) On the role of mean curvature in some singularly perturbed Neumann problems. SIAM J. Math. Anal. 31 (1), 63-79.

[4] Gierer, A. \& Meinhardt, H. (1972) A theory of biological pattern formation. Kybernetik (Berlin) 12, 30-39.

[5] Gilbarg, D. \& Trudinger, N. S. (1983) Elliptic partial differential equations of second order (2nd ed.). Grundlehren der Mathematischen Wissenschaften [Fundamental Principles of Mathematical Sciences], 224. Springer-Verlag.

[6] LI, Y. Y. (1998) On a singularly perturbed equation with Neumann boundary condition. Comm. Partial Differential Equations 23 (3-4), 487-545.

[7] Lin, C. S., Ni, W. M. \& TAKAGI, I. (1988) Large amplitude stationary solutions to a chemotaxis systems. J. Diff. Eq. 72, 1-27.

[8] NishiuRA, Y. (1982) Global structure of bifurcating solutions of some reaction-diffusion systems. SIAM J. Math. Anal. 13, 559-593.

[9] NI, W. M. (1998) Diffusion, cross-diffusion, and their spike-layer steady states. Not. AMS 45 (1), 9-18.

[10] Ni, W. M. \& TAKagi, I. (1991) On the shape of least energy solution to a semilinear Neumann problem. Comm. Pure. Appl. Math. 41, 819-851.

[11] Ni, W. M. \& TAKagI, I. (1993) Locating the peaks of least energy solutions to a semilinear Neumann problem. Duke. Math. J. 70, 247-281.

[12] Ni, W. M. \& TaKagi, I. (1995) Point condensation generated by a reaction-diffusion system in axially symmetric domains. Japan J. Indust. Appl. Math. 12, 327-365.

[13] Ni, W. M., TAKagi, I. \& Yanagida, E. (2001) Stability of least energy patterns of the shadow system for an activator-inhibitor model. Japan J. Indust. Appl. Math. 18, 259-272.

[14] Takagi, I. (1986) Point-condensation for a reaction-diffusion system. J. Diff. Eq. 61, 208-249.

[15] WeI, J. (1997) On the boundary spike layer solutions to a singularly perturbed neumann problem. J. Diff. Eq. 134, 104-133.

[16] WeI, J. (1999) On single interior spike solutions of the Gierer-Meinhardt system: uniqueness and spectrum estimate. Euro. J. Appl. Math. 10, 353-378.

[17] WeI, J. (2001) Uniqueness and critical spectrum of boundary spike solutions. Proc. Roy. Soc. Edinburgh Sect. A 131 (6), 1457-1480.

[18] Ward, M. J. \& WeI, J. (2002) Asymmetric spike patterns for the one-dimensional GiererMeinhardt model: equilibria and stability. Euro. J. Appl. Math. 13, 283-320.

[19] WARD, M. J. \& WeI, J. (2003) Hopf bifurcations and oscillatory instabilities of spike solutions for the one-dimensional Gierer-Meinhardt model. J. Nonlinear Sci. 13, 209-264.

[20] Ward, M. J. \& WeI, J. (2003) Hopf bifurcations of spike solutions for the shadow GiererMeinhardt model. Euro. J. Appl. Math. 14, 677-771. 